\title{
Heavy-quark spin and flavor symmetry partners of the $X(3872)$ revisited: What can we learn from the one boson exchange model?
}

\author{
Ming-Zhu Liu, ${ }^{1}$ Tian-Wei Wu, ${ }^{1}$ Manuel Pavon Valderrama, ${ }^{1,2, *}$ Ju-Jun Xie, ${ }^{3}$ and Li-Sheng Geng ${ }^{1,2, \dagger}$ \\ ${ }^{1}$ School of Physics and Nuclear Energy Engineering, Beihang University, Beijing 100191, China \\ ${ }^{2}$ International Research Center for Nuclei and Particles in the Cosmos and Beijing Key Laboratory of \\ Advanced Nuclear Materials and Physics, Beihang University, Beijing 100191, China \\ ${ }^{3}$ Institute of Modern Physics, Chinese Academy of Sciences, Lanzhou 730000, China
}

(Received 1 March 2019; published 15 May 2019)

\begin{abstract}
Heavy-quark symmetry as applied to heavy hadron systems implies that their interactions are independent of their heavy-quark spin (heavy-quark spin symmetry) and heavy flavor contents (heavy flavor symmetry). In the molecular hypothesis the $X(3872)$ resonance is a $1^{++} D^{*} \bar{D}$ bound state. If this is the case, the application of heavy-quark symmetry to a molecular $X(3872)$ suggests the existence of a series of partner states, the most obvious of which is a possible $2^{++} D^{*} \bar{D}^{*}$ bound state for which the two-body potential is identical to that of the $1^{++} D^{*} \bar{D}$ system, the reason being that these two heavy hadronantihadron states have identical light-spin content. As already discussed in the literature, this leads to the prediction of a partner state at $4012 \mathrm{MeV}$, at least in the absence of other dynamical effects which might affect the location of this molecule. However the prediction of further heavy-quark symmetry partners cannot be made solely on the basis of symmetry and requires additional information. We propose to use the one-boson-exchange model to fill this gap, in which case we will be able to predict or discard the existence of other partner states. Besides the isoscalar $2^{++} D^{*} \bar{D}^{*}$ bound state, we correctly reproduce the location and quantum numbers of the isovector hidden-bottom $Z_{b}(10610)$ and $Z_{b}(10650)$ molecular candidates. We also predict the hidden-bottom $1^{++} B^{*} \bar{B}^{*}$ and $2^{++} B^{*} \bar{B}^{*}$ partners of the $X(3872)$, in agreement with previous theoretical speculations, plus a series of other states. The isoscalar, doubly charmed $1^{+} D D^{*}$ and $D^{*} D^{*}$ molecules, and their doubly bottomed counterparts are likely to bind, providing a few instances of explicitly exotic systems.
\end{abstract}

DOI: 10.1103/PhysRevD.99.094018

\section{INTRODUCTION}

Hadronic molecules were conjectured four decades ago from a direct analogy to the deuteron and the nuclear forces that bind it $[1,2]$. The idea is that heavy hadrons can exchange light mesons-such as the pion, the sigma, the rho, and the omega - in the same way that nucleons do. In a few cases the interaction might be strong enough to bind the hadrons into a molecule [3-5]. This conjecture has become particularly relevant after the experimental discovery of the $X(3872)$ by the Belle Collaboration [6], which is usually considered to be a $1^{++} D^{*} \bar{D}$ bound state [7-9]. Besides the closeness of the $X(3872)$ to the open charm threshold $D^{0} D^{0 *}$, the most convincing evidence of the

\footnotetext{
mpavon@buaa.edu.cn

lisheng.geng@buaa.edu.cn
}

Published by the American Physical Society under the terms of the Creative Commons Attribution 4.0 International license. Further distribution of this work must maintain attribution to the author(s) and the published article's title, journal citation, and DOI. Funded by SCOAP. molecular nature of the $X(3872)$ is its isospin-breaking decays into $J / \Psi 2 \pi$ and $J / \Psi 3 \pi$ [10], which are easily explained in the molecular picture [11,12], but not if the $X(3872)$ is a more compact object [13]. However a direct confirmation of the molecular nature of the $X(3872)$ requires precise measurements of its $D^{0} \bar{D}^{0} \gamma$ and $D^{0} \bar{D}^{0} \pi^{0}$ decays $[8,14,15]$. The discovery of the $X(3872)$ was preceded by the detection of the (probably molecular) $D_{s 0}^{*}(2317) / D_{s 1}(2460)$ mesons [16,17], and was followed by the observation of other molecular candidates, in particular, the $Z_{b}$ 's $[18,19], Z_{c}$ 's $\left(Z_{c}(3900)\right.$ [20,21], $Z_{c}(4020)$ [22,23]), and $P_{c}(4450)$ [24], which was recently discovered to consist of two nearby peaks [the $P_{c}(4440)$ and $\left.P_{c}(4457)\right]$ and to have a lighter partner [the $P_{c}$ (4312)] [25].

Most of the known molecular candidates are suspected to be bound states of heavy hadrons, i.e., hadrons containing a heavy quark. As a consequence their interactions are constrained by heavy-quark symmetry. This by itself is able to explain a few interesting properties of the spectrum of the molecular candidates [26-32]. Heavy-quark symmetry can be divided into heavy-quark spin symmetry 
(HQSS: the interactions among heavy hadrons are independent of heavy-quark spin), heavy flavor symmetry (HFS: the interactions among heavy hadrons are independent of the heavy-quark flavor), and heavy antiquarkdiquark symmetry (a heavy antiquark behaves as a heavy diquark pair). In a few instances heavy-quark symmetry can be used to predict the existence of unobserved partners of known molecular states. From a molecular $X(3872)$ it is possible to deduce the existence of the $X(4012)$ or $X_{2}$ $[29,30]-\mathrm{a} 2^{++} D^{*} \bar{D}^{*}$ partner of the $X(3872)$-and a series of triply heavy pentaquark-like molecules [32]. From heavy-quark symmetry and the assumption that the $P_{c}$ (4450) [24] is a $J^{P}=\frac{3}{2}^{-} \bar{D}^{*} \Sigma_{c}$ molecule, we expect the existence of a $\frac{5}{2}^{-} \bar{D}^{*} \Sigma_{c}^{*}$ partner state-which we can call the $P_{c}(4515)$ in reference to its expected mass-plus a few triply heavy hexaquark-like molecules [33]. Now with the discovery of the new $P_{c}(4312)$ and the two-peak structure of the $P_{c}$ (4450) [25] we can effectively determine the seven possible $S$-wave heavy meson-baryon molecules [34,35], including the previously mentioned $\frac{5}{2} \bar{D}^{*} \Sigma_{c}^{*}$ state. Yet, with the exception of the doubly charmed pentaquark family, heavy-quark symmetry alone is in general not able to determine the full molecular spectrum by itself and has to be supplemented with additional information about hadron dynamics.

The one-boson-exchange (OBE) model [36,37], besides having played a central role in the seminal speculations about the existence of hadron molecules, is able to provide this missing information about the hadron-hadron interactions. In this model the potential between two hadrons is the consequence of the exchange of a series of light mesons $(\pi, \sigma, \rho$, and $\omega)$ that provide the necessary dynamics for binding. The OBE model is not completely free of ambiguities, though: for making concrete predictions, a form factor and a cutoff are required to regularize the unphysical short-range behavior of the light mesons. If we limit ourselves to qualitative predictions, then it is not necessary to determine $\Lambda$ : it will be enough to have $\Lambda \sim 1 \mathrm{GeV}$, i.e., the natural scale for light hadrons. But if we want quantitative predictions the specific choice of the cutoff $\Lambda$ is important. One of the observations we make in this work is that the cutoff $\Lambda$ can be effectively determined from the condition of reproducing the binding energy of a known molecular candidate, e.g., the X(3872). After determining the cutoff $\Lambda$ with this condition, we can explore how the OBE model applies to the particular cases of the heavy meson-meson and heavy meson-antimeson systems and what predictions are to be expected. This framework is an adaptation of the renormalized $O B E$ ideas of Ref. [38], which also represents the most important conceptual innovation of the present work with respect to previous applications of the OBE model to heavy hadron molecules; see Refs. [39-41] as representative examples. We find that the twin hidden-bottom isovector $Z_{b}(10610)$ and $Z_{b}(10650)$ resonance- $Z_{b}$ and $Z_{b}^{\prime}$ for short-is correctly reproduced by the OBE model. The hiddenbottom partner of the $X(3872)$ is also predicted in agreement with the previous literature, from Törnqvist [4] onwards. A series of additional molecular states are predicted, particularly in the hidden-bottom sector, which we will discuss later.

The manuscript is structured as follows. In Sec. II we review the application of heavy-quark spin symmetry to the heavy meson-antimeson system. In Sec. III we present the details of the one-boson-exchange model as applied to the heavy meson-antimeson system. In Sec. IV we determine the cutoff in the OBE model from the condition of reproducing the $X(3872)$ as a hadronic molecule, from which we predict in turn the full spectrum of heavy mesonantimeson molecules. Finally, we present our conclusions in Sec. V.

\section{HEAVY-QUARK SPIN SYMMETRY}

We first review the consequences of HQSS for heavy meson molecules. The quark content of the heavy mesons is $Q \bar{q}$. If the heavy-light quark-antiquark pair is in the $S$-wave, the total angular momentum of the heavy meson is $J=0,1$. The $J=0$ and $J=1$ mesons are denoted as $P$ and $P^{*}$, respectively. The fields of the $P$ and $P^{*}$ heavy mesons can be combined into a single (heavy-quark spin symmetric) superfield [42], the nonrelativistic version of which is

$$
H=\frac{1}{\sqrt{2}}\left[P+\vec{P}^{*} \cdot \vec{\sigma}\right],
$$

where $H$ is a $2 \times 2$ matrix and $\vec{\sigma}$ refers to the Pauli matrices. From the nonrelativistic superfield $H$ we can easily construct the contact-range Lagrangian for the heavy mesonmeson interaction. If we consider interactions that do not contain derivatives of the heavy meson fields, the most general Lagrangian will be [26]

$$
\begin{aligned}
\mathcal{L}_{4 H}= & C_{a} \operatorname{Tr}\left[H^{\dagger} H\right] \operatorname{Tr}\left[H^{\prime \dagger} H^{\prime}\right] \\
& +C_{b} \operatorname{Tr}\left[H^{\dagger} \sigma_{i} H\right] \operatorname{Tr}\left[H^{\prime \dagger} \sigma_{i} H^{\prime}\right],
\end{aligned}
$$

where we use $H$ and $H^{\prime}$ to denote heavy mesons of different flavor. Notice that we are ignoring isospin or flavor quantum numbers in the Lagrangian above. This is actually very powerful, because without HQSS there will be a total of six independent $S$-wave interactions, which are reduced to two. If we particularize for a heavy meson-antimeson pair $H=Q \bar{q}$ and $H^{\prime}=\bar{H}=\bar{Q} q$, we are required to have well-defined $C$ parity. In this case, the contact-range nonrelativistic potential for the $S$-wave reads

$$
\begin{gathered}
V\left(0^{++}, P \bar{P}\right)=C_{a}, \\
V\left(1^{++}, P^{*} \bar{P}\right)=C_{a}+C_{b},
\end{gathered}
$$




$$
\begin{gathered}
V\left(1^{+-}, P^{*} \bar{P}\right)=C_{a}-C_{b}, \\
V\left(0^{++}, P^{*} \bar{P}^{*}\right)=C_{a}-2 C_{b}, \\
V\left(1^{+-}, P^{*} \bar{P}^{*}\right)=C_{a}-C_{b}, \\
V\left(2^{++}, P^{*} \bar{P}^{*}\right)=C_{a}+C_{b} .
\end{gathered}
$$

This potential contains two interesting patterns. The first pattern is that the $1^{+-} P^{*} \bar{P}$ and $P^{*} \bar{P}^{*}$ potentials are identical, which implies that these two molecules should have the same binding energy. This might explain why the $Z_{c}, Z_{c}^{\prime}$ and $Z_{b}, Z_{b}^{\prime}$ resonances come in pairs $[28,43]$ (if they happen to be molecules). The second pattern is the $1^{++} P^{*} \bar{P}$ and $2^{++} P^{*} \bar{P}^{*}$ potentials, which are identical. According to this pattern, if the $1^{++} D^{*} \bar{D}$ system binds then the $2^{++} D^{*} \bar{D}^{*}$ should also bind. In particular, if the $X(3872)$ is a molecule, there should be a $2^{++}$molecule with a similar binding energy $[29,30]$. This molecule, the $X(4012)$, has not been observed experimentally yet and remains theoretical. Besides, other theoretical models predict the $2^{++}$partner of the $X(3872)$ to have a different mass that is not necessarily close to the $D^{*} \bar{D}^{*}$ threshold $[44,45]$.

The interesting thing about the contact-range Lagrangian of Eq. (2) is that it is all we need to describe heavy meson(anti)meson molecules with a reasonable degree of accuracy. The reason for this is that the Lagrangian of Eq. (2) can be interpreted as the leading-order Lagrangian of an effective field theory (EFT) for heavy meson interactions [29]. Within the EFT framework the heavy meson interaction is divided into a long-range and a short-range part. The short-range part contains everything with a range of the order of $1 / M$ (with $M=0.5-1.0 \mathrm{GeV}$, the typical hadronic energy scale) or shorter, while the long-range part contains interactions with a range larger than that value. From this definition the long-range piece only contains pion exchanges, while the short-range piece represents the exchange of all other light mesons $(\sigma, \rho, \omega)$, the contribution of which can be effectively encapsulated in a contact-range Lagrangian. It happens that for heavy meson-(anti)meson systems pion exchanges are usually subleading, i.e., their impact in the description of these systems is not as important as the Lagrangian of Eq. (2).

This leaves the contact-range Lagrangian of Eq. (2) as the leading-order EFT for heavy meson molecules. However the EFT description has a problem: there are two free parameters, or four once we consider that the different isospin channels have different couplings. The EFT framework does not provide information about the couplings $C_{a}$ and $C_{b}$, which have to be determined from existing physical information. For instance, if the $X(3872)$ is indeed an isoscalar $1^{++} D^{*} \bar{D}$ molecule, from the condition of reproducing the binding energy of the $X(3872)$ we can determine the linear combination

$$
V_{X}=C_{0 a}+C_{0 b},
$$

where we have added a subscript to the couplings to indicate the isospin channel: $C_{I a}$ and $C_{I b}$ with $I=0$ for the $X(3872)$. Analogously, if the $Z_{b}$ and $Z_{b}^{\prime}$ are isovector $1^{+-} B^{*} \bar{B}$ and $B^{*} \bar{B}^{*}$ molecules, we can determine another combination of couplings

$$
V_{Z}=C_{1 a}-C_{1 b}
$$

in exactly the same way (notice that we did not mention the $Z_{c}$ 's because their potential is identical to that of the $Z_{b}$ 's owing to HFS [32]).

Here lies the problem we want to address in this manuscript: besides the $X(3872)$ and the $Z_{b}$ 's there are no other clear molecular candidates. We simply cannot fix the four leading-order couplings and determine the full spectrum of heavy meson-antimeson molecules. For that reason we have to resort to a phenomenological model if we want to effectively predict the heavy meson molecular spectrum. The phenomenological model we will use here is the OBE model.

\section{THE ONE-BOSON-EXCHANGE MODEL}

In this section we explain the OBE model as applied to the heavy meson molecules. In the OBE model, the interaction between two hadrons is a direct consequence of the exchange of light mesons. In its most basic version these light mesons are the pion, the $\sigma$, the $\rho$, and the $\omega$. The OBE potential provided the first accurate description of the nuclear force [36,37], and the seminal idea for the first conjectures about the existence of heavy hadron molecules [1]. The OBE potential has its limitations too and there have been frequent discussions about the coupling constants of the mesons, in particular regarding the short-range piece of the OBE potential which is usually dominated by $\rho$ and $\omega$ exchange. From SU(3)-flavor symmetry and the OZI rule we expect $g_{\omega N N} \simeq 3 g_{\rho N N}$, but a good description of the nuclear scattering data usually requires $g_{\omega N N}>3 g_{\rho N N}$. Nowadays, owing to the conceptual frameworks provided by renormalization and effective field theory, we understand that these problems are derived from the fine-tuning of the nuclear forces; see Ref. [38] for a lucid exposition. Yet, the application of the OBE potential to hadronic molecules is in part less problematic because of its exploratory character.

\section{A. The Lagrangian}

If we use the nonrelativistic superfield $H$ defined in Eq. (1), we can write the interaction Lagrangian between the heavy and light mesons as follows: 


$$
\begin{gathered}
\mathcal{L}_{H H \pi}=-\frac{g}{\sqrt{2} f_{\pi}} \operatorname{Tr}\left[H^{\dagger} \vec{\sigma} \cdot \nabla(\vec{\tau} \cdot \vec{\pi}) H\right], \\
\mathcal{L}_{H H \sigma}=g_{\sigma} \operatorname{Tr}\left[H^{\dagger} \sigma H\right], \\
\mathcal{L}_{H H \rho}=g_{\rho} \operatorname{Tr}\left[H^{\dagger} \vec{\tau} \cdot \vec{\rho}^{0} H\right] \\
-\frac{f_{\rho}}{4 M} \epsilon_{i j k} \operatorname{Tr}\left[H^{\dagger} \sigma_{k} \vec{\tau} \cdot\left(\partial_{i} \vec{\rho}_{j}-\partial_{j} \vec{\rho}_{i}\right) H\right], \\
\mathcal{L}_{H H \omega}=-g_{\omega} \operatorname{Tr}\left[H^{\dagger} \omega^{0} H\right] \\
+\frac{f_{\omega}}{4 M} \epsilon_{i j k} \operatorname{Tr}\left[H^{\dagger} \sigma_{k}\left(\partial_{i} \omega_{j}-\partial_{j} \omega_{i}\right) H\right] .
\end{gathered}
$$

In the equations above $\pi, \sigma, \rho_{\mu}=\left(\rho_{0}, \rho_{i}\right)$ and $\omega_{\mu}=$ $\left(\omega_{0}, \omega_{i}\right)$ represent the light meson fields, where there is a Lorentz index in the $\rho$ and $\omega$ fields as they are vector mesons. The axial coupling of the pion is denoted by $g, g_{\sigma}$ is the coupling to the $\sigma$ meson, while for the vector mesons we have two different couplings: $g_{V}$ and $f_{V}$, with $V=\rho, \omega$. The couplings $g_{V}$ and $f_{V}$ are the strength of the "electric-type" and "magnetic-type" interactions of the vector mesons, respectively. For the magnetic-type term we include a mass $M$, which is there to make $f_{V}$ dimensionless. We will set $M$ to be the $D$-meson mass: $M=m_{D}=1.87 \mathrm{GeV}$.

\section{B. The OBE potential}

The OBE potential is written as the sum of the contributions of the exchanged mesons $(\pi, \sigma, \rho$, or $\omega)$,

$$
V=\zeta V_{\pi}+V_{\sigma}+V_{\rho}+\zeta V_{\omega}
$$

where $\zeta= \pm 1$ is a sign which we use to distinguish between the heavy meson-meson and meson-antimeson cases. We use the convention

$$
\begin{aligned}
& \zeta=+1 \text { for } H \bar{H} \\
& \zeta=-1 \text { for } H H
\end{aligned}
$$

i.e., the sign is positive for the heavy meson-antimeson case, which is the most commonly studied case in the context of heavy hadron molecules. The contribution of each light meson in momentum space is

$$
\begin{gathered}
V_{\pi}(\vec{q})=\eta \vec{\tau}_{1} \cdot \vec{\tau}_{2} \frac{g^{2}}{2 f_{\pi}^{2}} \frac{\vec{a}_{1} \cdot \vec{q} \vec{a}_{2} \cdot \vec{q}}{\vec{q}^{2}+\mu_{\pi}^{2}}, \\
V_{\sigma}(\vec{q})=-\frac{g_{\sigma}^{2}}{\vec{q}^{2}+m_{\sigma}^{2}}, \\
V_{\rho}(\vec{q})=\vec{\tau}_{1} \cdot \vec{\tau}_{2}\left[\frac{g_{\rho}^{2}}{\vec{q}^{2}+m_{\rho}^{2}}-\eta \frac{f_{\rho}^{2}}{4 M^{2}} \frac{\left(\vec{a}_{1} \times \vec{q}\right) \cdot\left(\vec{a}_{2} \times \vec{q}\right)}{\vec{q}^{2}+\mu_{\rho}^{2}}\right],
\end{gathered}
$$

$$
V_{\omega}(\vec{q})=-\frac{g_{\omega}^{2}}{\vec{q}^{2}+m_{\omega}^{2}}+\eta \frac{f_{\omega}^{2}}{4 M^{2}} \frac{\left(\vec{a}_{1} \times \vec{q}\right) \cdot\left(\vec{a}_{2} \times \vec{q}\right)}{\vec{q}^{2}+\mu_{\omega}^{2}}
$$

where $\eta= \pm 1$ is a sign, which we will define later, and $\vec{a}_{1}$ and $\vec{a}_{2}$ are vectors that depend on whether we are considering the $P \bar{P}, P^{*} \bar{P} / P \bar{P}^{*}$, or $P^{*} \bar{P}^{*}$ systems. The convention for $a_{i}$ (where $i=1,2$ is the vertex) is the following:

$$
\begin{gathered}
\vec{a}_{i}=0 \quad \text { for a } P \rightarrow P \text { vertex, } \\
\vec{a}_{i}=\vec{\epsilon}_{i} \quad \text { for a } P \rightarrow P^{*} \text { vertex, } \\
\vec{a}_{i}=\vec{\epsilon}_{i}^{*} \quad \text { for a } P^{*} \rightarrow P \text { vertex }, \\
\vec{a}_{i}=\vec{S}_{i} \quad \text { for a } P^{*} \rightarrow P^{*} \text { vertex },
\end{gathered}
$$

where $\vec{\epsilon}_{i}$ is the polarization vector of the $P^{*}$ meson and $\vec{S}_{i}$ are the spin- 1 matrices. The convention for the sign $\eta$ is different depending on whether we are in the heavy mesonmeson or heavy meson-antimeson system. For the heavy meson-meson case we have

$$
\begin{gathered}
\eta=+1 \quad \text { for the } P^{*} P+P^{*} P \text { potential, } \\
\eta=-1 \quad \text { for the } P^{*} P-P^{*} P \text { potential, } \\
\eta=+1 \quad \text { for the } P^{*} P^{*} \text { potential, }
\end{gathered}
$$

depending on whether we have a symmetric or antisymmetric $P P^{*}$ configuration. For the heavy meson-antimeson case we have

$$
\begin{gathered}
\eta=+1 \quad \text { for the } C=(-1)^{L} P^{*} \bar{P} \text { potential, } \\
\eta=-1 \quad \text { for the } C=(-1)^{L+1} P^{*} \bar{P} \text { potential, } \\
\eta=+1 \quad \text { for the } P^{*} \bar{P}^{*} \text { potential, }
\end{gathered}
$$

where $C$ refers to the $C$ parity of the heavy mesonantimeson system and $L$ refers to the orbital angular momentum. Notice that for the piece of the potential that is multiplied by $\eta$ we do not use the mass $m$ of the light meson, but rather the effective mass $\mu$ which is defined as

$$
\begin{gathered}
\mu^{2}=m^{2}-\Delta_{Q}^{2} \quad \text { for the } P P^{*} / P^{*} P \text { case } \\
\mu^{2}=m^{2} \quad \text { for the } P^{*} P^{*} \text { case }
\end{gathered}
$$


where for $Q=c$ we have that $\Delta_{c}=m_{D}^{*}-m_{D}$ is the mass splitting between the $D$ and $D^{*}$ charmed mesons. The reason is that these potentials imply a vertex in which the $P$ heavy meson transitions into a $P^{*}$ heavy meson and vice versa for the other vertex. For the vector mesons the difference between $\mu$ and $m$ in the $P P^{*} / P^{*} P$ potential is really small, and we will simply take the approximations $\mu_{\rho} \simeq m_{\rho}$ and $\mu_{\omega} \simeq m_{\omega}$. For the pion and the charmed mesons $D$ and $D^{*}$ we have instead that $m_{\pi} \simeq \Delta_{c}$. In this case we will make the simplification $\mu_{\pi}=0$ for the $D D^{*} / D^{*} D$ potential.

The coordinate-space potential is obtained from Fourier transforming the potentials of Eqs. (18)-(21), in which case we arrive at

$$
\begin{gathered}
V_{\pi}(\vec{r})=-\eta \vec{\tau}_{1} \cdot \vec{\tau}_{2} \frac{g^{2}}{6 f_{\pi}^{2}}\left[-\vec{a}_{1} \cdot \vec{a}_{2} \delta(\vec{r})+\vec{a}_{1} \cdot \vec{a}_{2} \mu_{\pi}^{3} W_{Y}\left(\mu_{\pi} r\right)\right. \\
\left.+S_{12}(\vec{r}) \mu_{\pi}^{3} W_{T}\left(\mu_{\pi} r\right)\right], \\
V_{\sigma}(\vec{r})=-g_{\sigma}^{2} m_{\sigma} W_{Y}\left(m_{\sigma} r\right),
\end{gathered}
$$

$$
\begin{aligned}
V_{\rho}(\vec{r})= & \vec{\tau}_{1} \cdot \vec{\tau}_{2}\left[g_{\rho}^{2} m_{\rho} W_{Y}\left(m_{\rho} r\right)+\eta \frac{f_{\rho}^{2}}{4 M^{2}}\left(-\frac{2}{3} \vec{a}_{1} \cdot \vec{a}_{2} \delta(\vec{r})\right.\right. \\
& \left.\left.+\frac{2}{3} \vec{a}_{1} \cdot \vec{a}_{2} \mu_{\rho}^{3} W_{Y}\left(\mu_{\rho} r\right)-\frac{1}{3} S_{12}(\hat{r}) \mu_{\rho}^{3} W_{T}\left(\mu_{\rho} r\right)\right)\right],
\end{aligned}
$$

$$
\begin{aligned}
V_{\omega}(\vec{r})= & -g_{\omega}^{2} m_{\omega} W_{Y}\left(m_{\omega} r\right)-\eta \frac{f_{\omega}^{2}}{4 M^{2}}\left(-\frac{2}{3} \vec{a}_{1} \cdot \vec{a}_{2} \delta(\vec{r})\right. \\
& \left.+\frac{2}{3} \vec{a}_{1} \cdot \vec{a}_{2} \mu_{\omega}^{3} W_{Y}\left(\mu_{\omega} r\right)-\frac{1}{3} S_{12}(\hat{r}) \mu_{\omega}^{3} W_{T}\left(\mu_{\omega} r\right)\right),
\end{aligned}
$$

where the functions $W_{Y}(x)$ and $W_{T}(x)$ are defined as

$$
\begin{gathered}
W_{Y}(x)=\frac{e^{-x}}{4 \pi x}, \\
W_{T}(x)=\left(1+\frac{3}{x}+\frac{3}{x^{2}}\right) \frac{e^{-x}}{4 \pi x} .
\end{gathered}
$$

\section{Form factors}

The momentum-space potentials of Eqs. (18)-(21) are computed under the assumption of point-like particles. The finite size of the mesons can be modeled by means of a form factor,

$$
V_{M}(\vec{q}, \Lambda)=V_{M}(\vec{q}) F^{2}(q, m, \Lambda),
$$

where the subscript $M=\pi, \sigma, \rho, \omega$ refers to the light meson that is being exchanged, $m$ refers to the mass of the exchanged meson, and $F$ refers to the form factor. Here we will use a multipolar parametrization of the type ${ }^{1}$

$$
F(q, m, \Lambda)=\left(\frac{\Lambda^{2}-m^{2}}{\Lambda^{2}-q^{2}}\right)^{n}
$$

where $q^{2}=q_{0}^{2}-\vec{q}^{2}$ is the 4-momentum of the exchanged meson and $n$ is the power of the multipolar form factor: for $n=1$ we have a monopolar form factor, for $n=2$ a dipolar one, etc. In principle, each of the light mesons can have a different form factor and cutoff, as happens in the OBE model as applied in the two-nucleon system. But this is only possible if there is plenty of experimental data to fit. This is not the case for hadronic molecules and thus we will simply choose to use the same form factor-a monopolar form factor $(n=1)$-and the same cutoff for each of the light mesons.

The inclusion of a form factor can be taken into account with the following changes in the coordinate-space potential:

$$
\begin{gathered}
\delta(r) \rightarrow m^{3} d(x, \lambda), \\
W_{Y}(x) \rightarrow W_{Y}(x, \lambda), \\
W_{T}(x) \rightarrow W_{T}(x, \lambda),
\end{gathered}
$$

with $\lambda=\Lambda / m$. For a monopolar form factor the functions $d, W_{Y}$, and $W_{T}$ read

$$
\begin{gathered}
d(x, \lambda)=\frac{\left(\lambda^{2}-1\right)^{2}}{2 \lambda} \frac{e^{-\lambda x}}{4 \pi}, \\
W_{Y}(x, \lambda)=W_{Y}(x)-\lambda W_{Y}(\lambda x)-\frac{\left(\lambda^{2}-1\right)}{2 \lambda} \frac{e^{-\lambda x}}{4 \pi}, \\
W_{T}(x, \lambda)=W_{T}(x)-\lambda^{3} W_{T}(\lambda x)-\frac{\left(\lambda^{2}-1\right)}{2 \lambda} \lambda^{2}\left(1+\frac{1}{\lambda x}\right) \frac{e^{-\lambda x}}{4 \pi} .
\end{gathered}
$$

For form factors of higher polarity we refer to the Appendix.

\footnotetext{
${ }^{1}$ This parametrization, which is the most commonly used for the OBE model, was put into question in Ref. [46] for the vector mesons. The reason is that in the hidden gauge formalism the Weinberg-Tomozawa terms are perfectly saturated from vectormeson exchange, but this only happens with a form factor that does not suppress the off-shell coupling of the vector mesons to hadrons. However, from the point of view of standard chiral perturbation theory, vector-meson exchange does not saturate the Weinberg-Tomozawa terms but rather their subleading corrections [47], in which case the off-shell suppression of a multipolar form factor becomes a very welcome feature.
} 


\section{Couplings}

The OBE potential depends on the pion axial coupling $g$, the sigma coupling $g_{\sigma}$, the vector meson electric- and magnetic-type couplings $g_{V}$ and $f_{V}$ with $V=\rho, \omega$, and the mass scale $M$. For the pion axial coupling we choose

$$
g=0.6
$$

which is compatible within errors with the experimental determination $g_{1}=0.59 \pm 0.01 \pm 0.07$ from the $D^{*} \rightarrow D \pi$ decay $[48,49]$. For the sigma coupling, we determine it from the nucleon-nucleon-sigma coupling in the nonlinear sigma model [50] $\left(g_{\sigma N N}=\sqrt{2} M_{N} / f_{\pi} \simeq 10.1\right)$ and the quark model [51] relation:

$$
g_{\sigma}=\frac{1}{3} g_{\sigma N N} \simeq 3.4 .
$$

From Sakurai's universality [52] we expect the electric-type $\rho$ coupling to be

$$
g_{\rho}=\frac{m_{\rho}}{2 f_{\pi}} \simeq 2.9,
$$

though this is merely a first approximation. For instance, Casalbuoni et al. [53] suggested instead

$$
g_{\rho}=\beta \frac{m_{\rho}}{2 f_{\pi}} \simeq 2.6,
$$

where $\beta=0.9$. We adopt this second estimation for the $\rho$ coupling, which is closer to the lattice QCD calculation of Ref. [54]: $g_{\rho}=2.6 \pm 0.1 \pm 0.4$ in the heavy-quark limit. For the magnetic-type $\rho$ coupling, we also follow Casalbuoni et al. [53] (who applied vector-meson dominance to the weak decays of the charmed mesons), in which case we obtain

$$
f_{\rho}=4 \lambda M \frac{m_{\rho}}{2 f_{\pi}} \simeq 11.7 \text { for } M=1.87 \mathrm{GeV},
$$

where $\lambda=0.6 \pm 0.1 \mathrm{GeV}^{-1}$. The apparently large value of $f_{\rho}$ is a consequence of taking $M$ equal to the $D$-meson mass, instead of a more natural scale. Finally, the couplings to the $\omega$ meson can be deduced from the ones of the $\rho$ meson, plus $\mathrm{SU}(3)$ flavor symmetry and the OZI rule, which lead us to

$$
g_{\omega}=g_{\rho} \quad \text { and } \quad f_{\omega}=f_{\rho} .
$$

Alternatively, these two relations can also be derived from writing the Lagrangian for the interaction between heavy and vector mesons with $\mathrm{SU}(3)$ flavor indices and the vector-meson nonet. A summary of the meson masses and the couplings we are using in this work can be found in Tables I and II.

\section{E. Wave functions and partial-wave projection}

The general wave function for a two-heavy-meson system takes the form

$$
|\Psi\rangle=\Psi_{J M}(\vec{r})\left|I M_{I}\right\rangle,
$$

where $\left|I M_{I}\right\rangle$ refers to the isospin wave function and $\Psi_{J M}$ refers to the spin and spatial wave function. For the isospin wave function we simply couple the isospin of the two particles; the only subtlety is the isospin convention for antiparticles (if we are dealing with a hadron-antihadron system), which can be found in Ref. [55] for the heavy antimeson case. The $\Psi_{J M}$ piece of the wave function can be written as a partial-wave sum,

$$
\left.\Psi_{J M}(\vec{r})=\left.\sum_{L S} \psi_{L S J}(r)\right|^{2 S+1} L_{J}\right\rangle .
$$

We use the spectroscopic notation ${ }^{2 S+1} L_{J}$ to denote a partial wave with total spin $S$, orbital angular momentum $L$, and total angular momentum $J$. The precise definition is

$$
\left.{ }^{2 S+1} L_{J}\right\rangle=\sum_{M_{S}, M_{L}}\left\langle L M_{L} S M_{S} \mid J M\right\rangle\left|S M_{S}\right\rangle Y_{L M_{L}}(\hat{r}),
$$

where $\left\langle L M_{L} S M_{S} \mid J M\right\rangle$ are the Clebsch-Gordan coefficients, $\left|S M_{S}\right\rangle$ is the spin wave function, and $Y_{L M_{L}}(\hat{r})$ are the spherical harmonics. For the $P P$ and $P \bar{P}$ system, the spin wave function is trivial,

$$
\left|S M_{S}(P P)\right\rangle=|00\rangle,
$$

as we are dealing with spin- 0 mesons. For the $P P^{*} / P^{*} P$ and $P \bar{P}^{*} / P^{*} \bar{P}$ system, only one of the heavy mesons has spin,

$$
\left|S M_{S}\left(P P^{*}\right)\right\rangle=\left|1 M_{S}\right\rangle .
$$

For the $P^{*} P^{*}$ and $P^{*} P^{*}$ systems, we have

$$
\begin{aligned}
\left|S M_{S}\left(P^{*} P^{*}\right)\right\rangle= & \sum_{M_{S 1}, M_{S 2}}\left\langle 1 M_{S 1} 1 M_{S 2} \mid S M_{S}\right\rangle \\
& \times\left|1 M_{S 1}\right\rangle\left|1 M_{S 2}\right\rangle,
\end{aligned}
$$

where $\left|1 M_{S 1}\right\rangle,\left|1 M_{S 2}\right\rangle$ are the spin wave functions of particles 1 and 2 .

The partial-wave projection of the OBE potential depends on the matrix elements of the $\vec{a}_{1} \cdot \vec{a}_{2}$ and $S_{12}$ operators, which are in turn independent of $J$ and $M$,

$$
\left\langle S^{\prime} L^{\prime} J^{\prime} M^{\prime}\left|\mathbf{O}_{12}\right| S L J M\right\rangle=\delta_{J J^{\prime}} \delta_{M M^{\prime}} \mathbf{O}_{S L, S^{\prime} L^{\prime}}^{J}
$$

where $\mathbf{O}_{12}=\vec{a}_{1} \cdot \vec{a}_{2}$ or $S_{12}$. The specific matrix elements of the spin-spin and tensor operators can be found in Table III 
for all of the molecular configurations that contain an $S$-wave (i.e., the ones that are more likely to bind).

\section{PREDICTIONS OF MOLECULAR STATES}

\section{A. The $X(3872)$ as a renormalization condition}

The predictions of the OBE potential depend on the form factor cutoff $\Lambda$. For a soft cutoff the OBE potential is too weak to form bound states, while for a hard cutoff the OBE potential is too strong, leading to overbinding or spurious bound states. The physical interpretation of the cutoff $\Lambda$ is that it represents the finite size of the hadrons. For the particular case of a multipolar form factor, the cutoff $\Lambda$ is expected to be larger than the masses of the exchanged light mesons but not considerably larger than the natural hadronic scale (about $1-2 \mathrm{GeV}$ ). ${ }^{2}$

For the heavy meson-antimeson system it is possible to uniquely determine $\Lambda$ from the mass of the $X(3872)$. For concreteness we will consider the $X(3872)$ in the isospinsymmetric limit, where it is an isoscalar $D^{*} \bar{D}$ bound state with positive $C$ parity $C=+1$ and a binding energy of about $4 \mathrm{MeV}$. Notice that the $4 \mathrm{MeV}$ figure comes from the difference between the location of the $X(3872)$ pole and the $D D^{*}$ threshold for the isospin-averaged masses of the charmed mesons. With this condition and the parameters of Tables I and II, the OBE potential generates the $X(3872)$ pole for the cutoff,

$$
\Lambda=\Lambda_{X}=1.01 \mathrm{GeV}
$$

which agrees with our expectations of a natural size cutoff. ${ }^{3}$ For comparison purposes, we notice that for the deuteron taken as a neutron-proton bound state with a binding energy $B_{2}=2.2 \mathrm{MeV}$ we obtain $\Lambda_{d}=0.86 \mathrm{GeV}$, which is of the same order of magnitude.

\section{B. Error estimations}

With the OBE potential and the cutoff determined from the renormalization condition, we are ready to compute the spectrum of heavy meson-(anti)meson molecules. The calculation of the spectrum will be affected by uncertainties that have to be estimated. The most important source of uncertainty is the OBE potential itself: with the exceptions of the pion and rho couplings, $g$ and $g_{\rho}$, the other couplings

\footnotetext{
${ }^{2}$ We notice that form factors of a higher polarity prefer larger values of $\Lambda$ : for a monopolar form factor the ideal cutoff is in the vicinity of $\Lambda \simeq 1 \mathrm{GeV}$, while for a dipolar form factor $\Lambda \simeq 1.5 \mathrm{GeV}$.

${ }^{3}$ In a previous work [55] we obtained $\Lambda=1.04_{-0.10}^{+0.18} \mathrm{GeV}$, which is a bit higher. The reason for the difference is that in Ref. [55] the effective pion mass for the one-pion-exchange piece of the OBE potential was taken to be $\mu_{\pi}=138 \mathrm{MeV}$ instead of $\mu_{\pi}=0$. It is interesting to notice that the difference is indeed small, which is consistent with the idea that one-pion exchange is perturbative, as expected in the charm meson-antimeson system from the analysis of Ref. [29].
}

TABLE I. Masses and quantum numbers of the light mesons of the OBE model $(\pi, \sigma, \rho, \omega)$ and the heavy mesons $\left(D, D^{*}, B, B^{*}\right)$.

\begin{tabular}{lcc}
\hline \hline Light meson & $I^{G}\left(J^{P C}\right)$ & $M(\mathrm{MeV})$ \\
\hline$\pi$ & $1^{-}\left(0^{-+}\right)$ & 138 \\
$\sigma$ & $0^{+}\left(0^{++}\right)$ & 600 \\
$\rho$ & $1^{+}\left(1^{--}\right)$ & 770 \\
$\omega$ & $0^{-}\left(1^{--}\right)$ & 780 \\
Heavy meson & $\left.I^{P}\right)$ & $M(\mathrm{MeV})$ \\
$D$ & $\frac{1}{2}\left(0^{-}\right)$ & 1867 \\
$D^{*}$ & $\frac{1}{2}\left(1^{-}\right)$ & 2009 \\
$B$ & $\frac{1}{2}\left(0^{-}\right)$ & 5279 \\
$B^{*}$ & $\frac{1}{2}\left(1^{-}\right)$ & 5325 \\
\hline \hline
\end{tabular}

TABLE II. Couplings of the light mesons of the OBE model $(\pi, \sigma, \rho, \omega)$ to the heavy meson fields. For the magnetic-type coupling of the $\rho$ and $\omega$ vector mesons we have used the decomposition $f_{V}=\kappa_{V} g_{V}$, with $V=\rho, \omega$. $M$ refers to the mass scale involved in the magnetic-type couplings.

\begin{tabular}{lc}
\hline \hline Coupling & Value for $P / P^{*}$ \\
\hline$g$ & 0.60 \\
$g_{\sigma}$ & 3.4 \\
$g_{\rho}$ & 2.6 \\
$g_{\omega}$ & 2.6 \\
$\kappa_{\rho}$ & 4.5 \\
$\kappa_{\omega}$ & 4.5 \\
$M$ & 1867 \\
\hline \hline
\end{tabular}

of the OBE potential are not particularly well known. For instance, $g_{\sigma}$ is derived from the quark model, for which there are no reliable error estimations, though $30 \%$ looks like a sensible figure; $g_{\omega}$ has been derived from $\mathrm{SU}(3)$ flavor symmetry and the OZI rule, where at least a $20 \%$ error is to be expected. These uncertainties will propagate into the calculation of the binding energies. Considering the error of each of the coupling constants separately is possible (by means of Monte Carlo techniques, for instance), but cumbersome. Instead, we will assign an overall relative error $\delta_{\mathrm{OBE}}$ for the $\mathrm{OBE}$ potential, that is,

$$
V=V_{\mathrm{OBE}}\left(1 \pm \delta_{\mathrm{OBE}}\right),
$$

where we take $\delta_{\mathrm{OBE}}=30 \%$. If we consider the $X$ channel, this will be the only error source we will consider, i.e.,

$$
V_{X}=V_{X, \mathrm{OBE}}\left(1 \pm \delta_{\mathrm{OBE}}\right)
$$

which obviously propagates into the determination of the cutoff $\Lambda_{X}$. In particular, we arrive at

$$
\Lambda_{X}=1.01_{-0.10}^{+0.18} \mathrm{GeV}
$$

Now if we consider molecular states different than the $X$, we notice that the OBE potential for these molecules has 
TABLE III. Matrix elements of the spin-spin and tensor operators for the partial waves we are considering in this work.

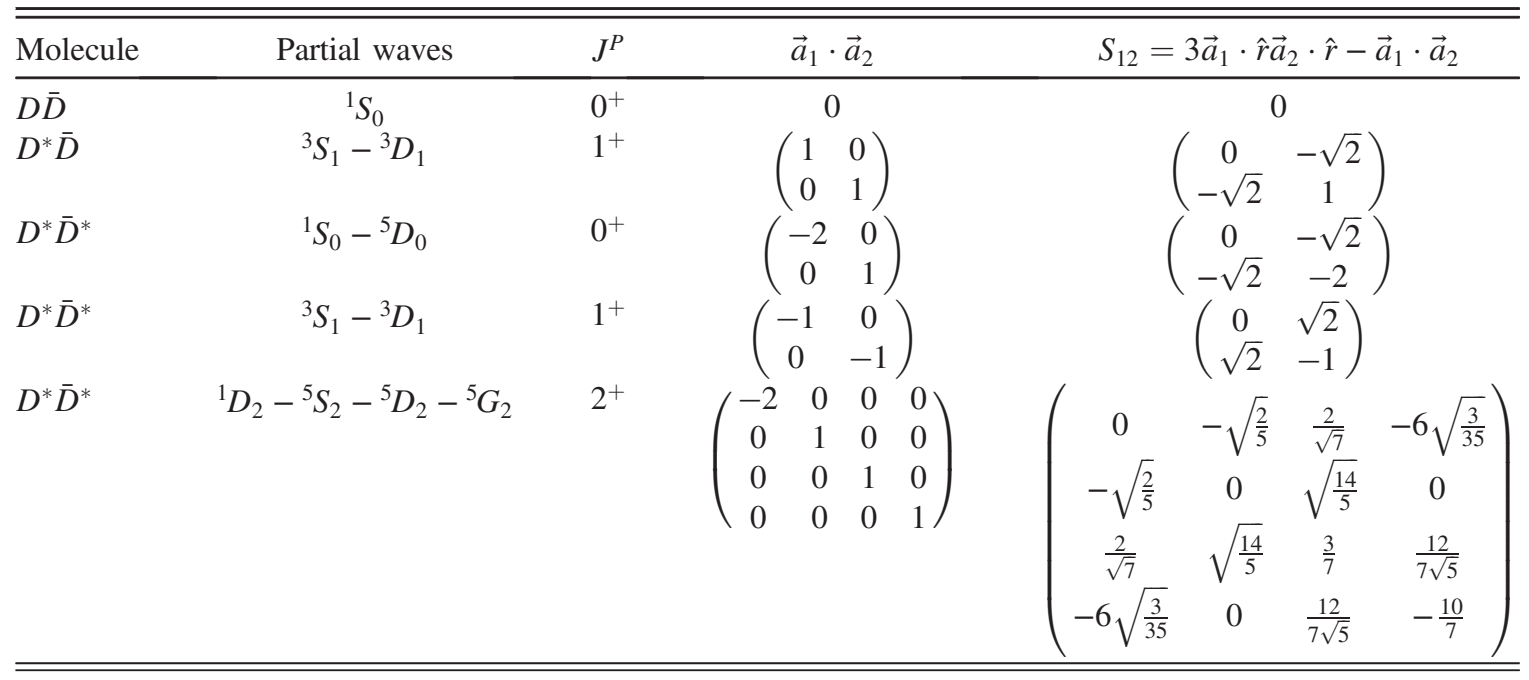

been derived from the assumption that heavy-quark symmetry applies. It happens that heavy-quark symmetry is not exact, but rather has an uncertainty. For that we include an additional, independent error source

$$
V=V_{\mathrm{OBE}}\left(1 \pm \delta_{\mathrm{OBE}}\right)\left(1 \pm \delta_{Q}\right)
$$

where $\delta_{Q}$ is the relative size of the expected violation of heavy-quark symmetry. In addition, the factor $\left(1 \pm \delta_{\mathrm{OBE}}\right)$ is identical to the one that we have previously taken for $V_{X}$. With this, the calculation of the binding energy is trivial: with $V$ and $\Lambda=\Lambda_{X}$ we determine the binding energy $B_{2}$ of the two-heavy-meson system. Finally, we sum in quadrature the two errors in $B_{2}$, the one propagated from $\left(1 \pm \delta_{\mathrm{OBE}}\right)$, and the second one propagated from $\left(1 \pm \delta_{Q}\right)$.

Besides the two-body binding energy $B_{2}$, we will also compute the scattering length $a_{2}$ of the two-meson systems. In the absence of bound states, the scattering length is useful to determine the strength of the interaction, particularly if it is close to binding. If we define the binding momentum as $\gamma_{2}=\sqrt{2 \mu B_{2}}$, where $\mu$ is the reduced mass of the two-body system and $B_{2}$ is its binding energy, we have the relation

$$
a_{2}=\frac{1}{\gamma_{2}}\left[1+\mathcal{O}\left(\frac{\gamma_{2}}{m_{\pi}}\right)\right],
$$

which is valid for $\gamma_{2} \ll m_{\pi}$, where we compare with the pion mass $m_{\pi}$ because it is the longest range contribution to the OBE potential. For $B_{2} \rightarrow 0$ the scattering length diverges: $a_{2} \rightarrow \infty$. When the bound state disappears but the attraction in the two-body system is still sizable, the scattering length will be large (with respect to the range of the pion) and negative: $a_{2}>-1 / m_{\pi} \sim-1.4 \mathrm{fm}$. We notice that for the $D^{*} \bar{D}$ and $D^{*} D$ systems the effective pion mass is close to zero, $\mu_{\pi} \simeq 0$. This in turn means that the tensor force from one-pion exchange effectively behaves as a $1 / r^{3}$ potential at large distance, which is known for not having a well-defined scattering length. For this reason we will not calculate the $D^{*} \bar{D}$ and $D^{*} D$ scattering lengths. For the calculation of the scattering length in settings with several coupled partial waves we refer to Ref. [56] which deals with the two-nucleon system, but the formalism therein can be easily translated to the two-heavy-meson system. The error estimations for the scattering length will be done exactly as those for the two-body binding energy.

Finally, we comment that there is another important source of (unknown) uncertainty in the calculation of $\Lambda_{X}$ : the impact of nearby charmonia. The $X(3872)$ is thought to be predominantly molecular as deduced from the isospinbreaking decays of the $X$ into $J / \Psi \pi^{+} \pi^{-}$and $J / \Psi \pi^{+} \pi^{-} \pi^{0}$ [10]. This branching ratio is naturally explained if the $X(3872)$ is molecular [11,12], but not if it is a charmonium state [13]. But the $X(3872)$ also decays into a charmonium and a photon, $\Gamma(X \rightarrow J / \Psi \gamma)$ and $\Gamma(X \rightarrow \Psi(2 S) \gamma)$ [57], which suggests the existence of $c \bar{c}$ components at shorter distances [58]. It is important to notice that only a small short-range $c \bar{c}$ component is required to explain the radiative charmonium decays [59], i.e., the $X(3872)$ is still mostly molecular [60]. It has also been argued that the coupled-channel dynamics between the $D^{*} \bar{D}$ degrees of freedom and the $\chi_{1}(2 P)$ charmonia might provide additional attraction to the system [61]. This additional attraction means that the actual form factor cutoff $\Lambda_{X}$ that is necessary to bind the $X(3872)$ might be a bit lower than the value we use here. However, it is difficult to estimate how much lower it is, as this depends on the $c \bar{c}$ probability for the $X$ (3872) [see also Ref. [62] for a more recent analysis of the degree of compositeness in the $X(3872)]$. For that reason we will not include this effect in our calculations and simply consider the $X(3872)$ to be a pure molecular state for simplicity. 


\section{The isoscalar heavy meson-antimeson system}

We begin by computing the spectrum for the isoscalar hidden charm and hidden bottom molecules. The use of the OBE potential [where the cutoff has been determined from the location of the $X(3872)]$ relies on two types of heavyquark symmetry: HQSS and HFS. The expected relative error of heavy-quark symmetry is $\delta_{Q} \sim \Lambda_{\mathrm{QCD}} / m_{Q}$, where $\Lambda_{\mathrm{QCD}} \sim 200-300 \mathrm{MeV}$. If we particularize for the charm quark mass, $m_{c} \sim 1.5 \mathrm{GeV}$, the error will be $\delta_{\mathrm{Q}} \sim 15 \%$. If we consider the hidden charm molecules, we will only make use of HQSS and the relative error $\delta_{Q}$ for the OBE potential will simply be $\delta_{Q}=\delta_{\mathrm{HQSS}} \sim 15 \%$. If we consider the hidden bottom molecules, we are actually using both HQSS and HFS: first, HQSS from applying the OBE potential for hidden charmed molecules different than the $X$, and then HFS from applying the same OBE potential in the hidden charm and hidden bottom sector. We account for this by adding the two errors in quadrature. The error comes from using the hidden charm sector as the starting point in the calculations, i.e., we take $\delta_{\mathrm{HQSS}}=\delta_{H F S}=\delta_{Q} \sim 15 \%$. Now, by adding both of these errors in quadrature we get $\delta_{Q}^{\prime}=\sqrt{2} \delta_{Q} \sim 20 \%$ for the hidden bottom sector.

With the OBE potential and the previous error estimations we arrive at the set of binding energies listed in Table IV. In the hidden charm sector we obtain that besides the $X(3872)$ the only other state that might survive is its $2^{++}$partner, the $X(4012)$ in reference to its expected mass, which was predicted in Refs. [29,30]. The uncertainty is large,

TABLE IV. Scattering lengths and binding energies of prospective isoscalar heavy meson-antimeson molecules. The error is a combination of the expected uncertainty of the OBE model and heavy-quark symmetry (HQSS and HFS). $M$ refers to the predicted mass (the central value) of a heavy meson molecule (if it binds).

\begin{tabular}{lccccc}
\hline \hline State & $I$ & $J^{P C}$ & $a_{2}(\mathrm{fm})$ & $B_{2}(\mathrm{MeV})$ & $M(\mathrm{MeV})$ \\
\hline$D \bar{D}$ & $0^{+}$ & $0^{++}$ & $-2.1_{-2.8}^{+1.7}$ & $\ldots$ & $\ldots$ \\
$D^{*} \bar{D}$ & $0^{+}$ & $1^{++}$ & N/A & 4 & 3872 \\
$D^{*} \bar{D}$ & $0^{+}$ & $1^{+-}$ & N/A & $\ldots$ & $\ldots$ \\
$D^{*} \bar{D}^{*}$ & $0^{+}$ & $0^{++}$ & $-1.5_{-1.0}^{+0.5}$ & $\ldots$ & $\ldots$ \\
$D^{*} \bar{D}^{*}$ & $0^{+}$ & $1^{+-}$ & $-2.0_{-2.7}^{+0.9}$ & $\ldots$ & $\ldots$ \\
$D^{*} \bar{D}^{*}$ & $0^{+}$ & $2^{++}$ & $+2.8_{-0.8}^{+4.5}$ & $4_{-6}^{+17}$ & 4013 \\
State & $I$ & $J^{P C}$ & $a_{2}(\mathrm{fm})$ & $B_{2}(\mathrm{MeV})$ & $M(\mathrm{MeV})$ \\
$B \bar{B}$ & $0^{+}$ & $0^{++}$ & $+1.3_{-0.5}^{+0.9}$ & $9_{-9}^{+13}$ & 10550 \\
$B^{*} \bar{B}$ & $0^{+}$ & $1^{++}$ & $+0.6 \pm 0.3$ & $51_{-38}^{+45}$ & 10553 \\
$B^{*} \bar{B}$ & $0^{+}$ & $1^{+-}$ & $+1.5_{-0.9}^{+1.2}$ & $7_{-7}^{+12}$ & 10595 \\
$B^{*} \bar{B}^{*}$ & $0^{+}$ & $0^{++}$ & $+1.2_{-1.2}^{+1.7}$ & $7_{-9}^{+21}$ & 10643 \\
$B^{*} \bar{B}^{*}$ & $0^{+}$ & $1^{+-}$ & $+1.5_{-0.9}^{+1.1}$ & $8_{-7}^{+12}$ & 10642 \\
$B^{*} \bar{B}^{*}$ & $0^{+}$ & $2^{++}$ & $+0_{-25}^{+1}$ & $59_{-43}^{+51}$ & 10591 \\
\hline \hline
\end{tabular}

$$
B_{2}\left(2^{++}, D^{*} \bar{D}^{*}\right)=4_{-6}^{+17},
$$

where the lower error (which is larger than the central value as a consequence of summing the OBE and heavy-quark symmetry uncertainties in quadrature) indicates that the state might very well disappear. Thus, the conclusion that the $X(4012)$ binds is not strong. In this sense the present work simply reaffirms the previous conclusions of Ref. [30], but with larger uncertainties owing to the uncertainty of using a phenomenological model instead of an EFT.

The existence of the $X(4012)$ has indeed been extensively discussed in the literature from different perspectives. Despite being a clear prediction of HQSS, the $X(4012)$ has not been experimentally observed yet. In principle it could be detected from $e^{+} e^{-} \rightarrow \psi(n S) \rightarrow \gamma X_{2}$ (with $\psi$ a $1^{--}$charmonium) in the $4.4-4.5 \mathrm{GeV}$ region [63]. If it is experimentally discarded in the future, a series of possible reasons for its disappearance have already been studied, such as the impact of nearby charmonia [61] or coupled-channel dynamics [45]. However, here we are inclined to favor the most simple explanation available: the natural uncertainty of HQSS in the charm sector is too large to guarantee the existence of the $X(4012)$.

If we consider the hidden bottom sector, we arrive at the conclusion that the six possible isoscalar molecules will bind. The prediction for the $X_{b 1}$, the hidden bottom partner of the $X(3872)$, is that its binding energy is

$$
B_{2}\left(X_{b 1}\right)=51_{-38}^{+45} \mathrm{MeV} .
$$

This is similar to the original calculation by Törnqvist [4], which used the one-pion-exchange potential with a monopolar form factor and a cutoff of $\Lambda=1.2 \mathrm{GeV}$, leading to $B_{2}\left(X_{b 1}\right) \sim 45 \mathrm{MeV}$. The seminal manuscript by Törnqvist is more exploratory than our calculations, as it considers only the longest range piece of the heavy meson-(anti) meson interaction (i.e., the one-pion-exchange potential). Despite these limitations, Törnqvist also predicted the other hidden bottom molecules that appear in Table IV, though in general the predicted binding energies are considerably larger than our results (with the exception of the $1^{++}$and $2^{++}$hidden bottom molecules, for which the predictions are similar). Of course, this is due to considering one-pion exchange only, in contrast to the exchange of other light mesons.

The comparison with the more recent calculation of Guo et al. [32] is more interesting: the authors of Ref. [32] used a contact-range effective field theory at leading order to predict the location of the $X_{b 1}$ and $X_{2 b}$ states, where the $X_{b 2}$ refers to the $2^{++} B^{*} \bar{B}^{*}$ molecule. The advantage of this approach is that EFT calculations are amenable to systematic error calculations, i.e., they are in principle more reliable than the phenomenological calculations we are using here. In this regard, it is interesting to check that the calculations of Ref. [32] predict that the $X_{b 1}$ and $X_{b 2}$ will 
TABLE V. Scattering lengths and binding energies of the prospective isovector heavy meson-antimeson molecules. Notice that the $Z_{b}(10610)$ and $Z_{b}(10650)$ molecular candidates are reproduced in the OBE model. The table is similar to Table IV, except for the following two details. (i) The $†$ symbol in the binding energy $B_{2}$ indicates here that the state disappears either from the OBE or the heavy-quark symmetry uncertainty alone. (ii) The $\pm \infty$ error in the scattering length $a_{2}$ indicates that the scattering length can cross infinity/minus infinity as a consequence of the appearance or disappearance of a bound state; when this happens, we include the expected bound of the scattering length in parentheses.

\begin{tabular}{lllccc}
\hline \hline State & $I^{G}$ & $J^{P C}$ & $a_{2}(\mathrm{fm})$ & $B_{2}(\mathrm{MeV})$ & $M(\mathrm{MeV})$ \\
\hline$D \bar{D}$ & $1^{-}$ & $0^{++}$ & $-0.7_{-0.4}^{+0.2}$ & $\ldots$ & $\ldots$ \\
$D^{*} \bar{D}$ & $1^{-}$ & $1^{++}$ & N/A & $\ldots$ & $\ldots$ \\
$D^{*} \bar{D}$ & $1^{+}$ & $1^{+-}$ & N/A & $\ldots$ & $\ldots$ \\
$D^{*} \bar{D}^{*}$ & $1^{-}$ & $0^{++}$ & $-1.9_{-2.5}^{+1.1}$ & $\ldots$ & $\ldots$ \\
$D^{*} \bar{D}^{*}$ & $1^{+}$ & $1^{+-}$ & $-1.1_{-0.5}^{+0.4}$ & $\ldots$ & $\ldots$ \\
$D^{*} \bar{D}^{*}$ & $1^{-}$ & $2^{++}$ & $-0.6 \pm 0.4$ & $\ldots$ & $\ldots$ \\
State & $I^{G}$ & $J^{P C}$ & $a_{2}(\mathrm{fm})$ & $B_{2}(\mathrm{MeV})$ & $M(\mathrm{MeV})$ \\
$B \bar{B}$ & $1^{-}$ & $0^{++}$ & $+4.6_{-3.3}^{+\infty(-24)}$ & $0_{\dagger}^{+4}$ & 10559 \\
$B^{*} \bar{B}$ & $1^{-}$ & $1^{++}$ & $-35_{-\infty(7)}^{+40}$ & $\ldots$ & $\ldots$ \\
$B^{*} \bar{B}$ & $1^{+}$ & $1^{+-}$ & $+1.7_{-0.4}^{+1.2}$ & $5_{-6}^{+11}$ & 10599 \\
$B^{*} \bar{B}^{*}$ & $1^{-}$ & $0^{++}$ & $+1.2_{-0.2}^{+0.4}$ & $15_{-15}^{+21}$ & 10635 \\
$B^{*} \bar{B}^{*}$ & $1^{+}$ & $1^{+-}$ & $+1.7_{-0.4}^{+1.1}$ & $5_{-6}^{+11}$ & 10645 \\
$B^{*} \bar{B}^{*}$ & $1^{-}$ & $2^{++}$ & $+28_{-24}^{+\infty(-5)}$ & $0_{\dagger}^{+1}$ & 10650 \\
\hline \hline
\end{tabular}

bind in the $B_{2} \sim 25-65 \mathrm{MeV}$ range, which is compatible with the results in Table IV. For a more complete comparison between the OBE model and heavy-meson EFT, we refer to Sec. IV G.

\section{The isovector heavy meson-antimeson system}

Next we consider the isovector hidden charm and hidden bottom molecules. The OBE potential leads to the spectrum of Table V, where we can appreciate that there are no hidden charm isovector molecules, while there are a few hidden bottom ones. In particular, the OBE potential predicts two twin $I^{G}\left(J^{P C}\right)=1^{+}\left(1^{+-}\right) B^{*} \bar{B}$ and $B^{*} \bar{B}^{*}$ molecules with a binding energy of

$$
B_{2}\left(1^{+-}, B^{(*)} \bar{B}\right)=5_{-6}^{+11} \mathrm{MeV} .
$$

Obviously, we are tempted to identify this prediction with the $Z_{b}(10610)$ and $Z_{b}(10650)$ resonances discovered by Belle $[18,19]$. Owing to their closeness to the $B^{*} \bar{B}$ and $B^{*} \bar{B}^{*}$ thresholds, the $Z_{b}$,s have been proposed to be molecular [43,64]. The analysis of Ref. [64] suggests a binding energy of $B_{2}=4.7_{-2.2}^{+2.3}$ and $0.11_{-0.06}^{+0.14} \mathrm{MeV}$ for the $Z_{b}$ and $Z_{b}^{\prime}$ respectively. The more recent analysis of
Ref. [65] suggests $B_{2}=0.9-1.7 \mathrm{MeV}$ for the $Z_{b}$ (i.e., a bit closer to threshold than in Ref. [64]), while the $Z_{b}^{\prime}$ could either be slightly bound $\left(B_{2} \sim 0.7 \mathrm{MeV}\right)$ or be a resonance just above the $B^{*} \bar{B}^{*}$ threshold. These numbers are indeed compatible with our results, which give a bit more confidence to the hypothesis that the $Z_{b}$ 's are molecular. Besides the $Z_{b}$ 's, there is another possible $B^{*} \bar{B}^{*}$ bound state for the quantum numbers $I^{G}\left(J^{P C}\right)=1^{-}\left(0^{++}\right)$with a binding energy of about $B_{2}=15 \mathrm{MeV}$ and there are two other configurations where the molecules might be close to the unitary limit, i.e., to having a bound state at threshold. These two configurations are the isovector $1^{-}\left(0^{++}\right) B \bar{B}$ and $1^{-}\left(2^{++}\right) B^{*} \bar{B}^{*}$ states. But the errors of these two predictions are sizable and it is impossible to determine their fate within the OBE model. For comparison purposes a recent work [66], which used the EFT formalism as applied to heavy meson-antimeson molecules, predicted that the six isovector hidden-bottom states will appear either as resonances above their respective two-meson thresholds for a pionful EFT or as virtual states for a pionless EFT (i.e., the overall picture is the same but the details are different).

The isovector hidden charm sector is interesting because the $Z_{c}(3900)$ and $Z_{c}(4020)$ resonances are usually regarded as probable molecular candidates. This is despite the fact that they are located a few $\mathrm{MeV}$ above the $D D^{*}$ and $D^{*} D^{*}$ thresholds, respectively, which is not the expected location for a standard $S$-wave bound state. Yet it is possible to interpret the $Z_{c}(3900)$ and $Z_{c}(4020)$ as resonances in $D D^{*}$ and $D^{*} D^{*}$ scattering. This hypothesis makes it natural to expect the $Z_{c}$ 's to be above threshold, but requires a potential that is repulsive at long distances and attractive at short distances, which is indeed the case. In the OBE potential for the isovector $J^{P C}=1^{+-} D \bar{D}^{*}$ and $D^{*} \bar{D}^{*}$ systems, the pion provides a repulsive long-range contribution and the sigma an attractive medium- and shortrange contribution. However, the $\rho$ and $\omega$ contributions cancel out perfectly in the limit where SU(3)-flavor symmetry and the OZI rule are exact. The $\rho$ and $\omega$ cancellation is problematic because it leads to a potential that is not strong enough to bind. In turn, this has prompted a few authors to consider the role of two-pion exchange in the $Z_{c}$ 's $[67,68]$ and $Z_{b}$ 's [69], a contribution which in the OBE model can be identified with the exchange of the $\sigma$ meson. Caution is advised, however: as argued in Ref. [70], the experimental information currently available might not be enough to determine whether the $Z_{c}$ 's are bound states, virtual states, or resonances. In Ref. [71] it was argued that both the bound and virtual state interpretations of the $Z_{c}(3900)$ are possible (with a slight preference for the virtual state). For this reason, what we will check is whether the interaction in the isovector $J^{P C}=1^{+-} D \bar{D}^{*}$ and $D^{*} \bar{D}^{*}$ systems is strong, for instance, by looking at the scatteringlength predictions. In Table $\mathrm{V}$ it can be appreciated that the scattering length for the $Z_{c}^{\prime}$ channels is $a_{2}=-1.1_{-0.5}^{+0.4} \mathrm{fm}$, while for the $Z_{c}$ the scattering length is not well defined in 
the $\mu_{\pi} \rightarrow 0$ limit we are taking. This scattering length might be compatible with the existence of a virtual state (which basically requires a large negative scattering length). Unfortunately, the magnitude of the scattering length is natural (of the order of the pion range), which means that this virtual state is probably not observable. According to this result, it is difficult to accommodate the $Z_{c}$ 's as pure molecular states.

This prompts us to consider the role of SU(3)-flavorsymmetry breaking in the formation of the $Z_{c}$ resonances. If $\mathrm{SU}(3)$-flavor symmetry is broken in the right direction, in particular by having $g_{\omega}>g_{\rho}$, this will generate an additional short-range attraction that might lead to a larger scattering length or to binding. We will consider the following three scenarios.

(1) Scenario A: SU(3)-flavor symmetry and the OZI rule are exactly respected, i.e., $g_{\omega}=g_{\rho}$ and $f_{\omega}=f_{\rho}$.

(2) Scenario B: $g_{\omega}=g_{\rho}$ and $f_{\omega}=f_{\rho}$ are moderately broken in the right way as to ease the binding of the $Z_{c}$ 's.

(3) Scenario C: Same as scenario B, but now the $g_{\omega}=$ $g_{\rho}$ and $f_{\omega}=f_{\rho}$ relations are strongly broken.

Scenario A simply corresponds to Table V, i.e., we follow the choice of coupling constants that we already made when discussing the OBE model. In scenario B we acknowledge that the $g_{\omega}=g_{\rho}$ and $f_{\omega}=f_{\rho}$ relations can be off by $\delta_{3}=20 \%$, while in scenario $\mathrm{C}$ the relations will be violated at the $\delta_{3}=35 \%$ level. The type of breakdown that makes the existence of the $Z_{c}$ 's as molecular states more probable is $g_{\omega}>g_{\rho}$ and $f_{\omega}>f_{\rho}$. In particular, we take

$$
\begin{array}{ll}
g_{\omega}^{\prime}=\left(1+\delta_{3}\right) g_{\mathrm{SU}(3)}, & g_{\rho}^{\prime}=\left(1-\delta_{3}\right) g_{\mathrm{SU}(3),} \\
f_{\omega}^{\prime}=\left(1+\delta_{3}\right) f_{\mathrm{SU}(3)}, & f_{\rho}^{\prime}=\left(1-\delta_{3}\right) f_{\mathrm{SU}(3)},
\end{array}
$$

where $g_{\mathrm{SU}(3)}$ and $f_{\mathrm{SU}(3)}$ refer to the previous values we were using for $g_{\rho / \omega}$ and $f_{\rho / \omega}$. With these values we have to calculate $\Lambda_{X}$ again, in which case we obtain

$$
\Lambda_{X}^{\prime}=1.03_{-0.10}^{+0.20} \mathrm{GeV}
$$

which is curiously identical for the $\delta_{3}=20 \%$ and $35 \%$ scenarios and very close to the original $\Lambda_{X}$ in the SU(3)symmetric limit; see Eq. (64) [this happens because the attraction lost from the $\rho$ in the $X(3872)$ channel is canceled out by the attraction gained from the $\omega]$. From $\Lambda_{X}^{\prime}$ we can recalculate the full spectrum of isoscalar hidden charm and hidden bottom molecules, in which case we arrive at the results of Table VI. We notice that for scenarios $B$ and $C$ the scattering length in the $Z_{c}^{\prime}$ channel increases to $a_{2}^{(B)}=-1.7_{-1.4}^{+0.7}$ and $a_{2}^{(C)}=-2.3_{-3.2}^{+1.2} \mathrm{fm}$, respectively. The scattering lengths cannot be excluded to be large once we
TABLE VI. Scattering lengths and binding energies of prospective isoscalar heavy meson-antimeson molecules if the $\rho$ and

\begin{tabular}{|c|c|c|c|c|c|c|}
\hline State & Scenario & $I^{G}$ & $J^{P C}$ & $a_{2}(\mathrm{fm})$ & $B_{2}(\mathrm{MeV})$ & $M(\mathrm{MeV})$ \\
\hline$D \bar{D}$ & $\mathrm{~B}$ & $1^{-}$ & $0^{++}$ & $-0.9_{-0.2}^{+0.3}$ & $\cdots$ & $\cdots$ \\
\hline$D^{*} \bar{D}$ & $\mathrm{~B}$ & $1^{-}$ & $1^{++}$ & N/A & $\cdots$ & $\cdots$ \\
\hline$D^{*} \bar{D}$ & $\mathrm{~B}$ & $1^{+}$ & $1^{+-}$ & N/A & $\cdots$ & $\cdots$ \\
\hline$D^{*} \bar{D}^{*}$ & B & $1^{-}$ & $0^{++}$ & $-4.4_{\infty(+36)}^{+2.7}$ & $\cdots$ & $\cdots$ \\
\hline$D^{*} \bar{D}^{*}$ & $\mathrm{~B}$ & $1^{+}$ & $1^{+-}$ & $-1.7_{-1.4}^{+0.7}$ & $\cdots$ & $\cdots$ \\
\hline$D^{*} \bar{D}^{*}$ & $\mathrm{~B}$ & $1^{-}$ & $2^{++}$ & $-0.8 \pm 0.3$ & $\cdots$ & $\cdots$ \\
\hline State & Scenario & $I^{G}$ & $J^{P C}$ & $a_{2}(\mathrm{fm})$ & $B_{2}(\mathrm{MeV})$ & $M(\mathrm{MeV})$ \\
\hline$D \bar{D}$ & $\mathrm{C}$ & $1^{-}$ & $0^{++}$ & $-1.0_{-0.5}^{+0.3}$ & $\cdots$ & $\cdots$ \\
\hline$D^{*} \bar{D}$ & $\mathrm{C}$ & $1^{-}$ & $1^{++}$ & N/A & $\cdots$ & $\cdots$ \\
\hline$D^{*} \bar{D}$ & $\mathrm{C}$ & $1^{+}$ & $1^{+-}$ & N/A & $\cdots$ & $\cdots$ \\
\hline$D^{*} \bar{D}^{*}$ & $\mathrm{C}$ & $1^{-}$ & $0^{++}$ & $-12_{-\infty(+6)}^{+12}$ & $\cdots$ & $\cdots$ \\
\hline$D^{*} \bar{D}^{*}$ & $\mathrm{C}$ & $1^{+}$ & $1^{+-}$ & $-2.3_{-3.2}^{+1.2}$ & $\cdots$ & $\cdots$ \\
\hline$D^{*} \bar{D}^{*}$ & $\mathrm{C}$ & $1^{-}$ & $2^{++}$ & $-0.9 \pm 0.3$ & $\cdots$ & $\cdots$ \\
\hline State & Scenario & $I^{G}$ & $J^{P C}$ & $a_{2}(\mathrm{fm})$ & $B_{2}(\mathrm{MeV})$ & $M(\mathrm{MeV})$ \\
\hline$B \bar{B}$ & $\mathrm{~B}$ & $1^{-}$ & $0^{++}$ & $+2.5_{-0.9}^{+4.2}$ & $2_{-3}^{+6}$ & 10557 \\
\hline$B^{*} \bar{B}$ & $\mathrm{~B}$ & $1^{-}$ & $1^{++}$ & $+8_{-5}^{+\infty(-11)}$ & $0_{\dagger}^{+2}$ & 10604 \\
\hline$B^{*} \bar{B}$ & $\mathrm{~B}$ & $1^{+}$ & $1^{+-}$ & $+1.3_{-0.4}^{+0.5}$ & $10_{-10}^{+17}$ & 10594 \\
\hline$B^{*} \bar{B}^{*}$ & $\mathrm{~B}$ & $1^{-}$ & $0^{++}$ & $+1.0_{-0.3}^{+0.4}$ & $26_{-24}^{+32}$ & 10624 \\
\hline$B^{*} \bar{B}^{*}$ & $\mathrm{~B}$ & $1^{+}$ & $1^{+-}$ & $+1.3_{-0.4}^{+0.5}$ & $11_{-10}^{+16}$ & 10639 \\
\hline$B^{*} \bar{B}^{*}$ & $\mathrm{~B}$ & $1^{-}$ & $2^{++}$ & $+6_{-4}^{+\infty(-15)}$ & $0_{\dagger}^{+2}$ & 10650 \\
\hline State & Scenario & $I^{G}$ & $J^{P C}$ & $a_{2}(\mathrm{fm})$ & $B_{2}(\mathrm{MeV})$ & $M(\mathrm{MeV})$ \\
\hline$B \bar{B}$ & $\mathrm{C}$ & $1^{-}$ & $0^{++}$ & $+2.1_{-0.9}^{+4.2}$ & $3_{-4}^{+7}$ & 10556 \\
\hline$B^{*} \bar{B}$ & $\mathrm{C}$ & $1^{-}$ & $1^{++}$ & $+5_{-3}^{+\infty(-110)}$ & $1_{\dagger}^{+2}$ & 10603 \\
\hline$B^{*} \bar{B}$ & $\mathrm{C}$ & $1^{+}$ & $1^{+-}$ & $+1.1_{-0.3}^{+0.4}$ & $14_{-14}^{+20}$ & 10590 \\
\hline$B^{*} \bar{B}^{*}$ & $\mathrm{C}$ & $1^{-}$ & $0^{++}$ & $+0.9_{-0.4}^{+0.2}$ & $34_{-22}^{+39}$ & 10616 \\
\hline$B^{*} \bar{B}^{*}$ & $\mathrm{C}$ & $1^{+}$ & $1^{+-}$ & $+1.1_{-0.3}^{+0.4}$ & $14_{-14}^{+20}$ & 10636 \\
\hline$B^{*} \bar{B}^{*}$ & $\mathrm{C}$ & $1^{-}$ & $2^{++}$ & $+5_{-3}^{+86}$ & $1_{\dagger}^{+3.0}$ & 10649 \\
\hline
\end{tabular}
$\omega$ couplings break SU(3)-flavor symmetry. The table is similar to Tables IV and V.

consider the uncertainty, yet neither the $Z_{c}$ nor the $Z_{c}^{\prime}$ bind for sensible values of $\mathrm{SU}(3)$ breaking.

\section{E. The heavy meson-meson system}

For the heavy meson-meson system our results are listed in Table VII. The most notable predictions in the doubly charmed sector are the twin isoscalar $J^{P}=1^{+} D D^{*}$ and $D^{*} D^{*}$ bound states, which are predicted to have binding energies of

$$
\begin{aligned}
& B_{2}\left(1^{+}, D D^{*}\right) \simeq 3_{-4}^{+15} \mathrm{MeV} \\
& B_{2}\left(1^{+}, D^{*} D^{*}\right) \simeq 2_{-3}^{+13} \mathrm{MeV}
\end{aligned}
$$


TABLE VII. Scattering lengths and binding energies of prospective heavy meson-meson molecules. Owing to the requirement of symmetric wave functions, the spin and isospin of the molecules are constrained by the relation $(-1)^{I+S+L+1}=1$. The exception is the $D^{*} D / D D^{*}$ system, which can appear in both isospin configurations, though the potential is different for each one. The table is similar to Tables IV and V.

\begin{tabular}{lccccc}
\hline \hline State & $I^{G}$ & $J^{P}$ & $a_{2}(\mathrm{fm})$ & $B_{2}(\mathrm{MeV})$ & $M(\mathrm{MeV})$ \\
\hline$D D$ & $1^{+}$ & $0^{+}$ & $-0.4_{-0.2}^{+0.1}$ & $\ldots$ & $\ldots$ \\
$D^{*} D+D D^{*}$ & $1^{+}$ & $1^{+}$ & $\mathrm{N} / \mathrm{A}$ & $\ldots$ & $\ldots$ \\
$D^{*} D-D D^{*}$ & $0^{+}$ & $1^{+}$ & $\mathrm{N} / \mathrm{A}$ & $3_{-4}^{+15}$ & 3873 \\
$D^{*} D^{*}$ & $1^{+}$ & $0^{+}$ & $-0.4 \pm 0.2$ & $\ldots$ & $\ldots$ \\
$D^{*} D^{*}$ & $0^{+}$ & $1^{+}$ & $4_{-2}^{+100}$ & $2_{-3}^{+13}$ & 4015 \\
$D^{*} D^{*}$ & $1^{+}$ & $2^{+}$ & $-0.6_{-0.3}^{+0.4}$ & $\ldots$ & $\ldots$ \\
State & $I^{G}$ & $J^{P}$ & $a_{2}(\mathrm{fm})$ & $B_{2}(\mathrm{MeV})$ & $M(\mathrm{MeV})$ \\
$B B$ & $1^{+}$ & $0^{+}$ & $-4.5_{-\infty}^{+4.0}$ & $\ldots$ & $\ldots$ \\
$B^{*} B+B B^{*}$ & $1^{+}$ & $1^{+}$ & $+2.4_{-0.9}^{+7.6}$ & $2_{-3}^{+8}$ & 10602 \\
$B^{*} B-B B^{*}$ & $0^{+}$ & $1^{+}$ & $+0.5_{-0.8}^{+0.4}$ & $58_{-44}^{+55}$ & 10546 \\
$B^{*} B^{*}$ & $1^{+}$ & $0^{+}$ & $-1.7_{-1.3}^{+0.8}$ & $\ldots$ & $\ldots$ \\
$B^{*} B^{*}$ & $0^{+}$ & $1^{+}$ & $+0.5_{-0.8}^{+0.4}$ & $58_{-43}^{+56}$ & 10592 \\
$B^{*} B^{*}$ & $1^{+}$ & $2^{+}$ & $+2.4_{-0.9}^{+6.3}$ & $2_{-3}^{+9}$ & 10648 \\
\hline \hline
\end{tabular}

where the binding energies are almost identical as a consequence of HQSS. There have been speculations about the existence of a doubly charmed tetraquark-like state with the quantum numbers of the twin doubly charmed molecules we predict. In the quark model the location of the ground state of the isoscalar $J^{P}=1^{+} c c \bar{q} \bar{q}$ tetraquark configuration can vary considerably, being sometimes predicted below [72-75] and sometimes above [76-78] the $D D^{*}$ threshold. A recent work considered the two-pionexchange potential in the heavy meson-meson system [79], predicting the isoscalar $J^{P}=1^{+} D D^{*}$ to be bound by about $20 \mathrm{MeV}$. On the lattice this tetraquark state has been recently predicted to be $23 \pm 11 \mathrm{MeV}$ below the $D D^{*}$ threshold [75]. The OBE model prediction indeed reinforces the previous speculations, though it gives a prediction much closer to threshold.

Owing to HFS, we also predict twin isoscalar $1^{+} B B^{*}$ and $B^{*} B^{*}$ molecules with a binding energy of $58 \mathrm{MeV}$, which are the heavy flavor partners of the $D D^{*}$ and $D^{*} D^{*}$ isoscalar molecules we discussed in the previous paragraph. This is in comparison with Ref. [80], which predicted the isoscalar $J^{P}=1^{+} B B^{*}$ and $B^{*} B^{*}$ bound states to have binding energies of 13 and $24 \mathrm{MeV}$, respectively, which moderately violate HQSS for $S$-wave interactions according to which both states should have similar binding energies. This suggests that in Ref. [80], which used the two-pion exchange potential, binding was maybe due to the $S D$-wave transitions induced by the
TABLE VIII. Scattering lengths and binding energies of prospective heavy meson-meson molecules in the flavor-exotic charm-antibottom sector. The table is similar to Tables IV and V.

\begin{tabular}{lllccc}
\hline \hline State & $I$ & $J^{P}$ & $a_{2}(\mathrm{fm})$ & $B_{2}(\mathrm{MeV})$ & $M(\mathrm{MeV})$ \\
\hline$D B$ & 0 & $0^{+}$ & $+14_{-15}^{+\infty(-4)}$ & $0_{\dagger}^{+3}$ & 7147 \\
$D B^{*}$ & 0 & $1^{+}$ & $+14_{-14}^{+\infty(-4)}$ & $0_{\dagger}^{+3}$ & 7192 \\
$D^{*} B$ & 0 & $1^{+}$ & $+7_{-7}^{+\infty(-6)}$ & $0_{\dagger}^{+3}$ & 7288 \\
$D^{*} B^{*}$ & 0 & $0^{+}$ & $-7_{-\infty(+13)}^{+5}$ & $\ldots$ & $\ldots$ \\
$D^{*} B^{*}$ & 0 & $1^{+}$ & $+270_{-380}^{+\infty(-3)}$ & $\ldots$ & $\ldots$ \\
$D^{*} B^{*}$ & 0 & $2^{+}$ & $+1.4_{-0.3}^{+0.5}$ & $20_{-22}^{+33}$ & 7314 \\
State & $I$ & $J^{P}$ & $a_{2}(\mathrm{fm})$ & $B_{2}(\mathrm{MeV})$ & $M(\mathrm{MeV})$ \\
$D B$ & 1 & $0^{+}$ & $-1.5_{-1.1}^{+0.6}$ & $\ldots$ & $\ldots$ \\
$D B^{*}$ & 1 & $1^{+}$ & $-1.5_{-1.2}^{+0.6}$ & $\ldots$ & $\ldots$ \\
$D^{*} B$ & 1 & $1^{+}$ & $-1.7_{-1.7}^{+0.7}$ & $\ldots$ & $\ldots$ \\
$D^{*} B^{*}$ & 1 & $0^{+}$ & $+6_{-4}^{+\infty(-5)}$ & $0_{\dagger}^{+8}$ & 7334 \\
$D^{*} B^{*}$ & 1 & $1^{+}$ & $-6_{-\infty(+10)}^{+4}$ & $\ldots$ & $\ldots$ \\
$D^{*} B^{*}$ & 1 & $2^{+}$ & $-1.3_{-0.8}^{+0.5}$ & $\ldots$ & $\ldots$ \\
\hline \hline
\end{tabular}

tensor force. Recently, two lattice QCD calculations $[75,81]$ predicted the isoscalar $J^{P}=1^{+} u d \bar{b} \bar{b}$ tetraquark to be located at $143 \pm 34 \mathrm{MeV}$ [75] and $128 \pm 24 \pm$ $10 \mathrm{MeV}$ [81] below the $B B^{*}$ threshold, respectively. Besides these exotic doubly bottomed tetraquark-like molecules, there are two other shallow (isovector) molecules: a $J^{P}=1^{+} B B^{*}$ and a $2^{+} B^{*} B^{*}$ bound state (see Table VII for details).

\section{F. Systems with two different flavors}

Finally, we consider the $D B$ (charmed-antibottom) and $D \bar{B}$ (charmed-bottom) family of heavy meson molecules. The most interesting is the charm-antibottom sector, which we summarize in Table VIII. In particular, we predict a series of molecular candidates close to the unitary limit, i.e., with a scattering length considerably larger than the range of molecular interaction (the range of the pion): $m_{\pi} a_{2} \gg 1$. The possibility that the $D B, D B^{*}$, and $D^{*} B$ molecules might have unnaturally large scattering lengths/form a shallow bound state has been already theorized in Ref. [82] from a simple argument involving the heavyquark spin decomposition of the heavy meson-antimeson interaction. The present calculation confirms the suspicions of Ref. [82] independently and adds a few more charmantibottom molecules which are also close to the unitary limit: the isoscalar/isovector $J^{P}=0^{+} / 1^{+} D^{*} B^{*}$ molecules. The isoscalar charm-bottom sector is also interesting: it contains a possible $J^{P}=1^{+} D^{*} \bar{B}^{*}$ bound state, which is the HFS partner of the doubly charmed and doubly bottomed molecule predicted in Table IX. In addition, though the $D \bar{B}$, 
TABLE IX. Scattering lengths and binding energies of prospective heavy meson-meson molecules in the flavor-exotic charm-bottom sector. The table is similar to Tables IV and V.

\begin{tabular}{lccccc}
\hline \hline State & $I$ & $J^{P}$ & $a_{2}(\mathrm{fm})$ & $B_{2}(\mathrm{MeV})$ & $M(\mathrm{MeV})$ \\
\hline$D \bar{B}$ & $0^{+}$ & $0^{+}$ & $-5_{-\infty(+22)}^{+5}$ & $\ldots$ & $\ldots$ \\
$D \bar{B}^{*}$ & $0^{+}$ & $1^{+}$ & $-5_{-\infty(+21)}^{+5}$ & $\ldots$ & $\ldots$ \\
$D^{*} \bar{B}$ & $0^{+}$ & $1^{+}$ & $-8_{-\infty(+9)}^{+8}$ & $\ldots$ & $\ldots$ \\
$D^{*} \bar{B}^{*}$ & $0^{+}$ & $0^{+}$ & $+1.0_{-0.3}^{+0.1}$ & $69_{-65}^{+88}$ & 7265 \\
$D^{*} \bar{B}^{*}$ & $0^{+}$ & $1^{+}$ & $+1.4_{-0.4}^{+0.7}$ & $16_{-19}^{+33}$ & 7318 \\
$D^{*} \bar{B}^{*}$ & $0^{+}$ & $2^{+}$ & $-7_{-\infty(+21)}^{+5}$ & $\ldots$ & $\ldots$ \\
& & & & & \\
State & $I$ & $J^{P}$ & $a_{2}(\mathrm{fm})$ & $B_{2}(\mathrm{MeV})$ & $M(\mathrm{MeV})$ \\
$D \bar{B}$ & $1^{+}$ & $0^{+}$ & $-0.7 \pm 0.4$ & $\ldots$ & $\ldots$ \\
$D \bar{B}^{*}$ & $1^{+}$ & $1^{+}$ & $-0.7 \pm 0.4$ & $\ldots$ & $\ldots$ \\
$D^{*} \bar{B}$ & $1^{+}$ & $1^{+}$ & $-0.8_{-0.4}^{+0.5}$ & $\ldots$ & $\ldots$ \\
$D^{*} \bar{B}^{*}$ & $1^{+}$ & $0^{+}$ & $-0.6_{-0.1}^{+0.1}$ & $\ldots$ & $\ldots$ \\
$D^{*} \bar{B}^{*}$ & $1^{+}$ & $1^{+}$ & $-0.6_{-0.4}^{+0.2}$ & $\ldots$ & $\ldots$ \\
$D^{*} \bar{B}^{*}$ & $1^{+}$ & $2^{+}$ & $-1.7_{-3.4}^{+1.1}$ & $\ldots$ & $\ldots$ \\
\hline \hline
\end{tabular}

$D \bar{B}^{*}$, and $D^{*} \bar{B}$ and $2^{+} D^{*} \bar{B}^{*}$ systems do not bind, their scattering lengths are also remarkably large.

The reason why the appearance of large scattering lengths is particularly interesting is because of the possibility of finding a few hadronic systems where universality happens [83]. Universality is the idea that all two-body systems with large scattering lengths (in comparison with the characteristic range of their interaction) can be described in the same way. A really interesting aspect of universality manifests when we consider the type of three-body systems that are derived from universal twobody systems. Three-body systems in which the two-body subsystems are close to the unitary limit can in principle display the Efimov effect [84], i.e., the existence of a geometric tower of three-body bound states where the ratio of the binding energies of a bound state and the next excited state approaches a constant value. The Efimov effect has been extensively studied in molecular physics (for a recent review, see Ref. [85]), where it was experimentally confirmed for the first time with cesium atoms [86]. Efimov physics is also known to play an important role in nuclear physics [87], for instance in the description of the triton [88-90], halo nuclei [91,92], and maybe even in the Hoyle state [93]. Our results strongly indicate that the bottombottom-anticharm three-meson system probably is one of the best candidates to find an Efimov trimer in hadronic physics, as originally suggested in Ref. [82].

\section{G. Comparison with heavy-meson EFT}

The OBE potential is a model, by which we mean that it is not clear how to estimate the reliability of the predictions. In contrast, EFTs are systematically improvable and allow for reliable error estimations. Inspired models, like the OBE model, are phenomenologically successful. This success is not a matter of method, but rather the outcome of inspired choices of what to include in the model. Thus, it is not trivial to determine the theoretical error of the binding energies and scattering lengths that we have derived from the OBE model, except with a direct comparison to experimental data. As a matter of fact the comparison to experiment is there, with the $Z_{b}$ and $Z_{b}^{\prime}$ resonance being correctly postdicted by the OBE model, but we are nonetheless limited to these two examples.

There are additional ways to indirectly assess the reliability of the OBE model. One possibility is to compare the predictions of the OBE model with the ones derived from an EFT. For this we will compare with the EFT for heavy-meson molecules developed in Ref. [29], which has been used in a series of works about heavy-meson molecules [30,32]. The EFT of Ref. [29], which we will call "heavy-meson EFT," is a refinement of previous ideas, in particular the contact theory with HQSS of Ref. [26] and X-EFT [14]. The problem with heavy-meson EFT (or with any other EFT) is that systematicity comes at the price of predictive power: the EFT formulation of Ref. [29] contains four independent couplings for the contact-range potential. These couplings are free parameters within the EFT and have to be determined from experimental information, e.g., from the location of a known hadronic molecule. It happens that the number of promising heavy meson-antimeson candidates is limited to the $X(3872)$, the $Z_{c}$ 's, and the $Z_{b}$ 's. If this were not enough, the $Z_{c}$ 's and $Z_{b}$ 's are connected by means of HQSS and HFS: heavy-meson EFT predicts that their contact-range potentials are identical. For this reason, of the four parameters of the heavymeson EFT at leading order-namely, $C_{0 a}, C_{0 b}, C_{1 a}$, and $C_{1 b}$-only two combinations can be determined, which are

$$
V_{X}=C_{0 a}+C_{0 b} \quad \text { and } \quad V_{Z}=C_{1 a}-C_{1 b},
$$

from which a limited number of additional predictions can be made. By comparing these few predictions with the corresponding ones in the OBE model we can form a better idea about the reliability of the OBE model.

Another possibility for testing the reliability of the predictions is to compare the OBE model with itself, by which we mean to compare the predictions obtained with different form factors but the same renormalization condition. If we choose a dipolar form factor (instead of a monopolar one), the cutoff for which the $X(3872)$ pole is reproduced changes to

$$
\Lambda_{X}^{D}=1.41_{-0.15}^{+0.28} \mathrm{GeV},
$$

which is roughly $\sqrt{2}$ larger than the monopolar cutoff. ${ }^{4}$ If the changes of the binding energy predictions with the

\footnotetext{
${ }^{4}$ For the deuteron with a dipolar form factor we have $\Lambda_{d}^{D}=1.23 \mathrm{GeV}$, which is also a factor of $\sqrt{2}$ larger than with a monopolar cutoff.
} 
TABLE X. Comparison of the predictions of the OBE model with different form factors (monopolar and dipolar) and with heavy-meson EFT. $B_{\mathrm{OBE}}^{M}$ and $B_{\mathrm{OBE}}^{D}$ are the binding energies (in MeV) computed from the OBE model and the renormalization condition with a monopolar and dipolar form factor, respectively. $B_{\mathrm{EFT}}(\Lambda)$ is the binding energy (in $\mathrm{MeV}$ ) in heavy-meson EFT for a given cutoff $\Lambda$ (in GeV), as taken from Ref. [32]. For the cases in which the system does not bind-namely, the $Z_{c}$ and $Z_{c}^{\prime}$ channels and their charm-antibottom counterpart-we compute the scattering length instead.

\begin{tabular}{llccccc}
\hline \hline State & $I^{G}$ & $J^{P}$ & $B_{\mathrm{OBE}}^{M}$ & $B_{\mathrm{OBE}}^{D}$ & $B_{\mathrm{EFT}}(\Lambda=0.5)$ & $B_{\mathrm{EFT}}(\Lambda=1.0)$ \\
\hline$D^{*} \bar{D}$ & $0^{+}$ & $1^{++}$ & Input & Input & Input & Input \\
$D^{*} \bar{D}^{*}$ & $0^{+}$ & $2^{++}$ & $4_{-6}^{+17}$ & $4_{-5}^{+14}$ & $5_{-4}^{+5}$ & $5_{-5}^{+12}$ \\
$D^{*} B^{*}$ & $0^{+}$ & $2^{++}$ & $20_{-22}^{+33}$ & $17_{-17}^{+25}$ & $12_{-6}^{+7}$ & $26_{-16}^{+20}$ \\
$B^{*} \bar{B}$ & $0^{+}$ & $1^{++}$ & $51_{-38}^{+45}$ & $41_{-29}^{+36}$ & $24_{-9}^{+8}$ & $65_{-25}^{+27}$ \\
$B^{*} \bar{B}^{*}$ & $0^{+}$ & $2^{++}$ & $59_{-43}^{+51}$ & $46_{-32}^{+38}$ & $24_{-9}^{+8}$ & $66_{-25}^{+27}$ \\
State & $I^{G}$ & $J^{P}$ & $B_{\mathrm{OBE}}^{M}$ & $B_{\mathrm{OBE}}^{D}$ & $B_{\mathrm{EFT}}(\Lambda=0.5)$ & $B_{\mathrm{EFT}}(\Lambda=1.0)$ \\
$B^{*} \bar{B}$ & $1^{+}$ & $1^{+-}$ & $5_{-6}^{+11}$ & $4_{-5}^{+9}$ & Input & Input \\
$B^{*} \bar{B}^{*}$ & $1^{+}$ & $1^{+-}$ & $5_{-6}^{+11}$ & $5_{-5}^{+9}$ & $2.1 \pm 2.1$ & $2.1_{-2.1}^{+2.5}$ \\
State & $I^{G}$ & $J^{P}$ & $a_{\mathrm{OBE}}^{M}$ & $a_{\mathrm{OBE}}^{D}$ & $a_{\mathrm{EFT}}(\Lambda=0.5)$ & $a_{\mathrm{EFT}}(\Lambda=1.0)$ \\
$D^{*} B^{*}$ & $1^{+}$ & $1^{+-}$ & $-6_{-\infty(+10)}^{+4}$ & $-6_{-\infty}^{+5}(+10)$ & $-8_{-\infty}^{+7}(+16)$ & $-1.2 \pm 0.6$ \\
$D^{*} \bar{D}$ & $1^{+}$ & $1^{+-}$ & $\mathrm{N} / \mathrm{A}$ & $\mathrm{N} / \mathrm{A}$ & $-1.4_{-1.0}^{+0.8}$ & $-0.4 \pm 0.1$ \\
$D^{*} \bar{D}^{*}$ & $1^{+}$ & $1^{+-}$ & $-1.1_{-0.5}^{+0.4}$ & $-1.2_{-0.5}^{+0.4}$ & $-1.6_{-1.1}^{+1.1}$ & $-0.5_{-0.1}^{+0.2}$ \\
\hline \hline
\end{tabular}

dipolar form factor lie within the errors we have estimated (which they do), this will also point towards the reliability of the model.

The comparison with the EFT and the dipolar form factor predictions is shown in Table $\mathrm{X}$. The heavy-meson EFT predictions can be divided into two groups: the predictions derived from the existence of the $X(3872)$ and the ones derived from the $Z_{b}(10610)$. We denote which state has been used to determine the EFT couplings with the term "Input" in Table X. The comparison is actually very interesting: the predictions of the OBE model with a monopolar and dipolar form factor are compatible between themselves and with the ones from EFT within errors. Besides, the uncertainties of the OBE model predictions are in general larger than the EFT ones. This might indicate two things: (i) we have overestimated the OBE errors, though only by a small margin, or (ii) the EFT errors have been underestimated, as recently hypothesized in Ref. [94] based on the impossibility of formulating a cutoff-independent EFT compatible with HFS for heavy hadron molecules. Be that as it may, the similarity of the OBE and EFT predictions suggests an acceptable degree of reliability.

\section{DISCUSSION AND CONCLUSIONS}

We have considered the heavy meson-antimeson and heavy meson-meson systems with the following two assumptions: (i) the OBE model describes their interaction, and (ii) heavy-quark symmetry further constrains the dynamics of these systems. The physics of the OBE potential are intuitive and well-motivated, but there is the limitation that it requires a form factor and a cutoff for predictions to be possible. While the choice of form factor is not that important, the choice of a cutoff is crucial: without a way to reliably determine the cutoff it is not possible to make concrete predictions. To determine this cutoff we have used the assumption that the $X(3872)$ is indeed a $D^{*} \bar{D}$ bound state with quantum numbers $I=0$, $J^{P C}=1^{++}$and a binding energy of about $4 \mathrm{MeV}$ in the isospin-symmetric limit (where we used the isospinsymmetric limit for simplicity). From the cutoff determined with this renormalization condition, predictions in the $\mathrm{OBE}$ model are possible. We also included error estimations for these predictions.

By considering the isoscalar hidden charm sector, we found that besides the existence of the $X(3872)$ it is plausible to expect that the $X_{c 2}$-the $I=0, J^{P C}=2^{++}$ $D^{*} \bar{D}^{*}$ system-also binds. The expected binding energy of the $X_{c 2}$ is $B_{2}=4_{-6}^{+17} \mathrm{MeV}$, where the uncertainty is however too large to guarantee the existence of this HQSS partner of the $X(3872)$. The other isoscalar hidden charm molecules are not expected to bind, even after taking into account the uncertainty of the OBE model and HQSS. It is interesting to compare these results with previous explorations. The possible existence of the $X_{c 2}$ was already discussed in the seminal work of Törnqvist [4], which predicted an isoscalar $J^{P C}=1^{++} D \bar{D}^{*}$ bound state [presumably the $X(3872)]$ and pointed out that the $J^{P C}=2^{++}$ $D^{*} \bar{D}^{*}$ system was close to binding, requiring only a bit of extra attraction to bind. In Ref. [30] the full six possible HQSS partners of the $X(3872)$ were predicted, though this 
work indicated that the predictions depend on a series of assumptions, with some predictions being more reliable than others. In particular, Ref. [30] indicated that the most robust prediction is that of the $X_{2}$, which solely relies on the hypothesis that the $X(3872)$ is molecular. This is also what we found in our exploration. It is also worth mentioning the $I=0, J^{P C}=0^{++} D \bar{D}$ system, which according to theoretical explorations [30,95] could also form a shallow molecule, the $X(3700)$. Here we found a considerable amount of attraction in the isoscalar $D \bar{D}$ system, which has a negative scattering length $a=-2.1_{-2.8}^{+1.7} \mathrm{fm}$, but no binding within the uncertainties of the OBE model. But we did not consider coupled-channel effects, which mix the $0^{++} D \bar{D}$ and $D^{*} \bar{D}^{*}$ systems and lead to additional attraction. From EFT arguments, coupled-channel effects are expected to be a small correction for two-heavy-meson molecules [29], but the $D \bar{D}$ system is close to binding and small effects could make a difference. We did not consider coupled channels in this work, but we mention that a breaking of HQSS by $40 \%$ (which is not particularly probable statistically but not particularly improbable either) will lead to binding.

In the isoscalar hidden bottom sector the conclusion is that all six possible molecules can bind, with the $J^{P C}=1^{++}$and $2^{++}$molecules-the $X_{b 1}$ and $X_{b 2}$-being the most bound ones with $B_{2} \sim 50-60 \mathrm{MeV}$. We noticed that the first prediction of the $X_{b 1}$ and $X_{b 2}$-the hidden bottom partner of the $X(3872)$-already appeared in Törnqvist [4]. After this a series of theoretical works $[26,31,96,97]$ - including ours - have only reinforced this conclusion further. The only problem is that the $X_{b 1}$ has not been detected in experiments. In this regard, Karliner and Rosner [98] have suggested that the $\chi_{b 1}(3 P)$ (with a mass $M=10512 \mathrm{MeV}$ ) might not be a $J^{P C}=1^{++}$bottomonium after all, but rather the bottom partner of the $X(3872)$. The quantum numbers of the $\chi_{b 1}(3 P)$ indeed coincide with the $X_{b 1}$ and the required binding energy lies within the error estimations of the OBE model $(M=10508-10591 \mathrm{MeV})$.

The isovector hidden charm sector is also interesting owing to its connection with the $Z_{c}(3900)$ and $Z_{c}(4012)$ molecular candidates. The application of the OBE model with SU(3)-symmetric couplings leads to the conclusion that these two molecules do not bind but are probably virtual states instead, as deduced from the moderately large negative scattering length. This is the same conclusion as in Ref. [31]. We point out that even if the $Z_{c}(3900)$ and $Z_{c}$ (4012) are assumed to be molecular, it cannot be determined if they are genuine bound states, resonances, or virtual states from the experimental data; see Ref. [70] for details. In this regard the SU(3)-symmetric OBE model will be compatible with the virtual state hypothesis. If we allow for natural violations of SU(3)-flavor symmetry in the couplings, the situation is qualitatively the same as before: there is no binding within the expected theoretical uncertainties. However, we predicted larger scattering lengths than in the $\mathrm{SU}(3)$-symmetric limit, again pointing towards the virtual state hypothesis.

In the isovector hidden bottom sector the $Z_{b}$ 's are correctly postdicted as bound states, both of which have a binding energy of $B_{2}=5_{-6}^{+11} \mathrm{MeV}$. This figure is not far from other estimations of their binding energies, for instance the estimations based on the analysis of the experimental data done in Refs. $[64,65]$. Besides, finding the $Z_{b}$ 's in the OBE model further substantiates the idea that they have a sizable molecular component, as proposed in Refs. [43,64].

Regarding the doubly charmed sector, we found that the $I=0, J^{P}=1^{+} D^{*} D$ and $D^{*} D^{*}$ systems form molecules with binding energies of $B_{2}=3_{-4}^{+15}$ and $2_{-3}^{+13} \mathrm{MeV}$, respectively. This type of hadron with $c c \bar{q} \bar{q}$ quark content has indeed been predicted in the quark model [72-78] (as a compact tetraquark, however with large uncertainties regarding its location), in a molecular model that includes two-pion exchange [79] (with a binding energy of $B_{2}=20 \mathrm{MeV}$ ) and recently on the lattice [75], with $B_{2}=22 \pm 11 \mathrm{MeV}$. The HFS partners in the charmbottom $(c b \bar{q} \bar{q})$ and doubly bottom $(b b \bar{q} \bar{q})$ sectors were also predicted, with binding energies of $B_{2}=15_{-20}^{+30}$ and $60_{-50}^{+60} \mathrm{MeV}$, respectively. The first qualitative prediction of the $Q Q \bar{q} \bar{q}$ family of tetraquark-like molecules (where $Q=b, c$, i.e., a heavy quark) was made long ago by Manohar and Wise [99]. The recent lattice calculations of Refs. [75,81] suggest a binding energy for the isoscalar $J^{P}=1^{+}$bottom-bottom tetraquark of $143 \pm 34$ and $128 \pm$ $24 \pm 10 \mathrm{MeV}$ respectively (relative to the $B B^{*}$ thresholds), while the recent quark-model calculation of Ref. [100] located the isoscalar $J^{P}=1^{+}$charm-bottom tetraquark at $B_{2}=164 \mathrm{MeV}$ with respect to the $D^{*} \bar{B}^{*}$ threshold. Notice that here we have predicted a molecular state instead of a compact tetraquark.

Of particular interest is the charm-antibottom sector ( $c \bar{b} q \bar{q}$-type molecules), where a series of two-body states with large scattering lengths are predicted. This in turn points to the possibility of Efimov physics in the $B B D$, $B B D^{*}, B B^{*} D, B^{*} B^{*} D$, and $B^{*} B^{*} D^{*}$ three-body systems, as previously conjectured in Ref. [82]. Besides having a twobody subsystem close to the unitary limit, the family of bottom-bottom-charm three-body systems displays a moderate mass imbalance between the charm and bottom mesons, which is a factor that is known to enhance Efimov physics [101]. This family of three-hadron systems probably provides one of the most promising systems in which to observe Efimov trimers, which so far have only been observed in atomic systems. For this reason the exploration of the charm-antibottom sector, either experimentally or on the lattice, is a really interesting subject.

Finally, we have tried to determine the reliability of the OBE model as applied to heavy meson molecules. 
Models, in contrast to theories, are not amenable to error estimations that are fully systematic. For this reason it is of particular importance to carefully confront the OBE model predictions with other approaches. From the experimental point of view, the correct postdiction of the $Z_{b}$ 's indicates that the OBE model correctly describes the bulk of the physics of heavy meson molecules. From the theoretical point of view, we have compared a set of predictions derived from heavy-meson EFT with those of the OBE model. The agreement seems to indicate that the OBE model is reliable.

\section{ACKNOWLEDGMENTS}

We would like to thank Muhammad Naeem Anwar for comments. This work is partly supported by the National Natural Science Foundation of China under Grant No. 11735003, the Fundamental Research Funds for the Central Universities, the Youth Innovation Promotion Association CAS (No. 2016367), and the Thousand Talents Plan for Young Professionals.

\section{APPENDIX: MULTIPOLAR FORM FACTORS}

The OBE model generates a singular potential, where the tensor components of the potential diverge as $1 / r^{3}$ at short distances. This type of divergence is unphysical and can be regularized by means of a form factor. The most common type of form factor for the OBE model is the multipolar form factor we have written in Eq. (41), where depending on the exponent we talk about a monopolar $(n=1)$, dipolar $(n=2)$, etc. form factor. In principle the exponent $n$ can depend on the meson $M$, though here we will assume that all off the mesons have the same type of form factor. In general the contribution of a meson $M$ to the OBE potential will be obtained by Fourier transforming from momentum to coordinate space as

$$
V_{M}(\vec{r})=\int \frac{d^{3} \vec{q}}{(2 \pi)^{3}} V_{M}(\vec{q})\left(\frac{\Lambda^{2}-m^{2}}{\Lambda^{2}-q^{2}}\right)^{2 n}
$$

but as we have seen, this transformation is relatively direct once we take into account that the contribution of the form factor can be encapsulated by the substitutions

$$
\begin{gathered}
\delta(r) \rightarrow m^{3} d(x, \lambda ; 2 n), \\
W_{Y}(x) \rightarrow W_{Y}(x, \lambda ; 2 n), \\
W_{T}(x) \rightarrow W_{T}(x, \lambda ; 2 n),
\end{gathered}
$$

with $\lambda=\Lambda / m$ and where we have labeled them with $2 n$, i.e., with twice the polarity of the form factor for the exchanged meson. The function $d$ can be evaluated analytically for $k=2 n=1,2,3, \ldots$ (i.e., integer $k$ ), with

$$
d(x, \lambda ; 1)=\left(\lambda^{2}-1\right) \frac{e^{-\lambda x}}{4 \pi x},
$$

$d(x, \lambda ; k \geq 2)=\frac{i\left(\lambda^{2}-1\right)^{k}}{(k-1) ! 2^{k-1} \lambda^{2 k-3}}(i \lambda x)^{k-1} \frac{h_{k-2}^{(+)}(i \lambda x)}{4 \pi}$,

where $h_{n}^{( \pm)}(z)=j_{n}(z) \pm i y_{n}(z)$ are the Hankel spherical functions, which we have defined in terms of the Bessel spherical functions $j_{n}(z)$ and $y_{n}(z)$. For the function $W_{Y}$ we can evaluate it recursively as

$$
W_{Y}(x, \lambda ; 1)=W_{Y}(x)-\lambda W_{Y}(\lambda x),
$$

$$
W_{Y}(x, \lambda ; k \geq 2)=W_{Y}(x, \lambda ; k-1)-\frac{d(x, \lambda ; k)}{\lambda^{2}-1},
$$

while for $W_{T}$ we have

$$
W_{T}(x, \lambda ; 1)=W_{T}(x)-\lambda^{3} W_{T}(\lambda x)
$$

$W_{T}(x, \lambda ; 2)=W_{T}(x, \lambda ; 1)-\frac{\left(\lambda^{2}-1\right)}{2 \lambda} \lambda^{2}\left(1+\frac{1}{\lambda x}\right) \frac{e^{-\lambda x}}{4 \pi}$,

$W_{T}(x, \lambda ; 3)=W_{T}(x, \lambda ; 2)-\frac{\left(\lambda^{2}-1\right)^{2}}{8 \lambda}(\lambda x) \frac{e^{-\lambda x}}{4 \pi}$,

$$
\begin{aligned}
W_{T}(x, \lambda ; k \geq 4)= & W_{T}(x, \lambda ; k-1) \\
& -\frac{i\left(\lambda^{2}-1\right)^{k-1}}{(k-1) ! 2^{k-1} \lambda^{2 k-7}} x^{2}(i \lambda x)^{k-3} \frac{h_{k-4}^{(+)}(i \lambda x)}{4 \pi} .
\end{aligned}
$$

A monopolar form factor (on both vertices) corresponds to the $k=2$ solution, while a dipolar one corresponds to the $k=4$ solution. 
[1] M. Voloshin and L. Okun, JETP Lett. 23, 333 (1976).

[2] A. De Rujula, H. Georgi, and S. Glashow, Phys. Rev. Lett. 38, 317 (1977).

[3] N. A. Tornqvist, Phys. Rev. Lett. 67, 556 (1991).

[4] N. A. Tornqvist, Z. Phys. C 61, 525 (1994).

[5] T. E. O. Ericson and G. Karl, Phys. Lett. B 309, 426 (1993).

[6] S. K. Choi et al. (Belle Collaboration), Phys. Rev. Lett. 91, 262001 (2003).

[7] N. A. Tornqvist, arXiv:hep-ph/0308277.

[8] M. B. Voloshin, Phys. Lett. B 579, 316 (2004).

[9] E. Braaten and M. Kusunoki, Phys. Rev. D 69, 074005 (2004).

[10] S.-K. Choi, S. Olsen, K. Trabelsi, I. Adachi, H. Aihara et al., Phys. Rev. D 84, 052004 (2011).

[11] D. Gamermann and E. Oset, Phys. Rev. D 80, 014003 (2009).

[12] D. Gamermann, J. Nieves, E. Oset, and E. Ruiz Arriola, Phys. Rev. D 81, 014029 (2010).

[13] C. Hanhart, Yu. S. Kalashnikova, A. E. Kudryavtsev, and A. V. Nefediev, Phys. Rev. D 85, 011501 (2012).

[14] S. Fleming, M. Kusunoki, T. Mehen, and U. van Kolck, Phys. Rev. D 76, 034006 (2007).

[15] F. K. Guo, C. Hidalgo-Duque, J. Nieves, A. Ozpineci, and M. Pavon Valderrama, Eur. Phys. J. C 74, 2885 (2014).

[16] B. Aubert et al. (BABAR Collaboration), Phys. Rev. Lett. 90, 242001 (2003).

[17] D. Besson et al. (CLEO Collaboration), Phys. Rev. D 68, 032002 (2003); 75, 119908(E) (2007).

[18] A. Bondar et al. (Belle Collaboration), Phys. Rev. Lett. 108, 122001 (2012).

[19] A. Garmash et al. (Belle Collaboration), Phys. Rev. D 91, 072003 (2015).

[20] M. Ablikim et al. (BESIII Collaboration), Phys. Rev. Lett. 110, 252001 (2013).

[21] Z. Q. Liu et al. (Belle Collaboration), Phys. Rev. Lett. 110, 252002 (2013).

[22] M. Ablikim et al. (BESIII Collaboration), Phys. Rev. Lett. 111, 242001 (2013).

[23] M. Ablikim et al. (BESIII Collaboration), Phys. Rev. Lett. 113, 212002 (2014).

[24] R. Aaij et al. (LHCb Collaboration), Phys. Rev. Lett. 115, 072001 (2015).

[25] R. Aaij et al. (LHCb Collaboration), arXiv:1904.03947.

[26] M. T. AlFiky, F. Gabbiani, and A. A. Petrov, Phys. Lett. B 640, 238 (2006).

[27] M. Voloshin, Phys. Rev. D 84, 031502 (2011).

[28] T. Mehen and J. W. Powell, Phys. Rev. D 84, 114013 (2011).

[29] M. Pavon Valderrama, Phys. Rev. D 85, 114037 (2012).

[30] J. Nieves and M. Pavon Valderrama, Phys. Rev. D 86, 056004 (2012).

[31] F.-K. Guo, C. Hidalgo-Duque, J. Nieves, and M. Pavon Valderrama, Phys. Rev. D 88, 054007 (2013).

[32] F.-K. Guo, C. Hidalgo-Duque, J. Nieves, and M. Pavon Valderrama, Phys. Rev. D 88, 054014 (2013).

[33] M.-Z. Liu, F.-Z. Peng, M. Sánchez Sánchez, and M. Pavon Valderrama, Phys. Rev. D 98, 114030 (2018).
[34] M.-Z. Liu, Y.-W. Pan, F.-Z. Peng, M. Sánchez Sánchez, L.-S. Geng, A. Hosaka, and M. Pavon Valderrama, arXiv:1903.11560.

[35] C. W. Xiao, J. Nieves, and E. Oset, arXiv:1904.01296.

[36] R. Machleidt, K. Holinde, and C. Elster, Phys. Rep. 149, 1 (1987).

[37] R. Machleidt, Adv. Nucl. Phys. 19, 189 (1989).

[38] A. Calle Cordon and E. Ruiz Arriola, Phys. Rev. C 81, 044002 (2010).

[39] X. Liu, Z.-G. Luo, Y.-R. Liu, and S.-L. Zhu, Eur. Phys. J. C 61, 411 (2009).

[40] Z.-C. Yang, Z.-F. Sun, J. He, X. Liu, and S.-L. Zhu, Chin. Phys. C 36, 6 (2012).

[41] Z.-F. Sun, J. He, X. Liu, Z.-G. Luo, and S.-L. Zhu, Phys. Rev. D 84, 054002 (2011).

[42] A. F. Falk and M. E. Luke, Phys. Lett. B 292, 119 (1992).

[43] A. E. Bondar, A. Garmash, A. I. Milstein, R. Mizuk, and M. B. Voloshin, Phys. Rev. D 84, 054010 (2011).

[44] R. Molina and E. Oset, Phys. Rev. D 80, 114013 (2009).

[45] V. Baru, E. Epelbaum, A. A. Filin, C. Hanhart, U.-G. Meißner, and A. V. Nefediev, Phys. Lett. B 763, 20 (2016).

[46] A. Ozpineci, C. W. Xiao, and E. Oset, Phys. Rev. D 88, 034018 (2013).

[47] G. Ecker, J. Gasser, A. Pich, and E. de Rafael, Nucl. Phys. B321, 311 (1989).

[48] S. Ahmed et al. (CLEO Collaboration), Phys. Rev. Lett. 87, 251801 (2001).

[49] A. Anastassov et al. (CLEO Collaboration), Phys. Rev. D 65, 032003 (2002).

[50] M. Gell-Mann and M. Levy, Nuovo Cimento 16, 705 (1960).

[51] D. O. Riska and G. E. Brown, Nucl. Phys. A679, 577 (2001).

[52] J. J. Sakurai, Ann. Phys. (N.Y.) 11, 1 (1960).

[53] R. Casalbuoni, A. Deandrea, N. Di Bartolomeo, R. Gatto, F. Feruglio, and G. Nardulli, Phys. Lett. B 299, 139 (1993).

[54] W. Detmold, C. J. D. Lin, and S. Meinel, Phys. Rev. D 85, 114508 (2012).

[55] M.-Z. Liu, T.-W. Wu, J.-J. Xie, M. Pavon Valderrama, and L.-S. Geng, Phys. Rev. D 98, 014014 (2018).

[56] M. Pavon Valderrama and E. Ruiz Arriola, Phys. Rev. C 72, 044007 (2005).

[57] R. Aaij et al. (LHCb Collaboration), Nucl. Phys. B886, 665 (2014).

[58] E. S. Swanson, Phys. Lett. B 598, 197 (2004).

[59] Y. Dong, A. Faessler, T. Gutsche, and V. E. Lyubovitskij, J. Phys. G 38, 015001 (2011).

[60] F.-K. Guo, C. Hanhart, Yu. S. Kalashnikova, U.-G. Meißner, and A. V. Nefediev, Phys. Lett. B 742, 394 (2015).

[61] E. Cincioglu, J. Nieves, A. Ozpineci, and A. U. Yilmazer, Eur. Phys. J. C 76, 576 (2016).

[62] X.-W. Kang and J. A. Oller, Eur. Phys. J. C 77, 399 (2017).

[63] F.-K. Guo, U.-G. Meißner, and Z. Yang, Phys. Lett. B 740, 42 (2015).

[64] M. Cleven, F.-K. Guo, C. Hanhart, and U.-G. Meissner, Eur. Phys. J. A 47, 120 (2011).

[65] Q. Wang, V. Baru, A. A. Filin, C. Hanhart, A. V. Nefediev, and J. L. Wynen, Phys. Rev. D 98, 074023 (2018). 
[66] V. Baru, A. Epelbaum, A. A. Filin, C. Hanhart, A. V. Nefediev, and Q. Wang, arXiv:1901.10319.

[67] F. Aceti, M. Bayar, J. M. Dias, and E. Oset, Eur. Phys. J. A 50, 103 (2014).

[68] F. Aceti, M. Bayar, E. Oset, A. Martinez Torres, K. P. Khemchandani, J. M. Dias, F. S. Navarra, and M. Nielsen, Phys. Rev. D 90, 016003 (2014).

[69] J. M. Dias, F. Aceti, and E. Oset, Phys. Rev. D 91, 076001 (2015).

[70] M. Albaladejo, F.-K. Guo, C. Hidalgo-Duque, and J. Nieves, Phys. Lett. B 755, 337 (2016).

[71] Q.-R. Gong, Z.-H. Guo, C. Meng, G.-Y. Tang, Y.-F. Wang, and H.-Q. Zheng, Phys. Rev. D 94, 114019 (2016).

[72] J. Carlson, L. Heller, and J. A. Tjon, Phys. Rev. D 37, 744 (1988).

[73] B. A. Gelman and S. Nussinov, Phys. Lett. B 551, 296 (2003).

[74] J. Vijande, A. Valcarce, and N. Barnea, Phys. Rev. D 79, 074010 (2009).

[75] P. Junnarkar, N. Mathur, and M. Padmanath, Phys. Rev. D 99, 034507 (2019).

[76] M. Karliner and J. L. Rosner, Phys. Rev. Lett. 119, 202001 (2017).

[77] E. J. Eichten and C. Quigg, Phys. Rev. Lett. 119, 202002 (2017).

[78] T. Mehen, Phys. Rev. D 96, 094028 (2017).

[79] H. Xu, B. Wang, Z.-W. Liu, and X. Liu, Phys. Rev. D 99, 014027 (2019).

[80] B. Wang, Z.-W. Liu, and X. Liu, Phys. Rev. D 99, 036007 (2019).

[81] L. Leskovec, S. Meinel, M. Pflaumer, and M. Wagner, arXiv:1904.04197.

[82] M. Pavon Valderrama, Phys. Rev. D 98, 034017 (2018).

[83] E. Braaten and H. W. Hammer, Phys. Rep. 428, 259 (2006).
[84] V. Efimov, Phys. Lett. 33B, 563 (1970).

[85] P. Naidon and S. Endo, Rep. Prog. Phys. 80, 056001 (2017).

[86] T. Kraemer, M. Mark, P. Waldburger, J. G. Danzl, C. Chin, B. Engeser, A. D. Lange, K. Pilch, A. Jaakkola, H.-C. Nägerl, and R. Grimm, Nature (London) 440, 315 (2006).

[87] H.-W. Hammer and L. Platter, Annu. Rev. Nucl. Part. Sci. 60, 207 (2010).

[88] P. F. Bedaque, H. W. Hammer, and U. van Kolck, Phys. Rev. Lett. 82, 463 (1999).

[89] P. F. Bedaque, H. W. Hammer, and U. van Kolck, Nucl. Phys. A646, 444 (1999).

[90] P. F. Bedaque, H. W. Hammer, and U. van Kolck, Nucl. Phys. A676, 357 (2000).

[91] D. V. Federov, A. S. Jensen, and K. Riisager, Phys. Rev. Lett. 73, 2817 (1994).

[92] H. W. Hammer, C. Ji, and D. R. Phillips, J. Phys. G 44, 103002 (2017).

[93] H. W. Hammer and R. Higa, Eur. Phys. J. A 37, 193 (2008).

[94] V. Baru, E. Epelbaum, J. Gegelia, C. Hanhart, U. G. Meißner, and A. V. Nefediev, Eur. Phys. J. C 79, 46 (2019).

[95] D. Gamermann, E. Oset, D. Strottman, and M. Vicente Vacas, Phys. Rev. D 76, 074016 (2007).

[96] C.-Y. Wong, Phys. Rev. C 69, 055202 (2004).

[97] J. Nieves and M. Valderrama, Phys. Rev. D 84, 056015 (2011).

[98] M. Karliner and J. L. Rosner, Phys. Rev. D 91, 014014 (2015).

[99] A. V. Manohar and M. B. Wise, Nucl. Phys. B399, 17 (1993).

[100] T. F. Caramés, J. Vijande, and A. Valcarce, Phys. Rev. D 99, 014006 (2019).

[101] K. Helfrich, H. W. Hammer, and D. S. Petrov, Phys. Rev. A 81, 042715 (2010). 